\title{
Status of the NICA project at JINR
}

\author{
Alexei Sissakian and Alexander Sorin ${ }^{\mathrm{a}}$ \\ Bogoliubov Laboratory of Theoretical Physics, JINR Dubna, 141980 Dubna, Russia
}

\begin{abstract}
The status of the new flagship Project Nuclotron-based Ion Collider fAcility (NICA) at the Joint Institute for Nuclear Research, its main parameters, construction details and physics program is reported. The generation of intense heavy ion and polarized light nuclear NICA beams is aimed at searching for the phase transitions, mixed phase and critical phenomena of nuclear matter and investigation of polarization phenomena at the collision energies up to $\sqrt{s_{N N}}=11 \mathrm{GeV}$.
\end{abstract}

We would like to thank the NICA/MPD Project members for fruitful collaboration. Special thanks we express to D. Blaschke, A. Efremov, V. Kekelidze, I. Meshkov, G. Musulmanbekov, A. Nagaytsev, O. Rogachevsky, I. Savin, O. Shevchenko, V. Skokov, $\mathrm{O}$. Teryaev and V. Toneev for valuable discussions. This research was partly supported by the Russian Foundation for Basic Research, grant No. 08-02-01003-a.

\section{References}

1. A.N. Sissakian, A.S. Sorin, M.K. Suleymanov, V.D. Toneev, G.M. Zinovjev, Properties of strongly interacting matter and the search for a mixed phase at the JINR Nuclotron, Phys. Part. Nucl. Lett. 5 (2008) 1; nucl-ex/0511018, nucl-ex/0601034.

2. A.N. Sissakian, A.S. Sorin, V.D. Toneev, QCD Matter A Search for a Mixed Quark-Hadron Phase, Proceedings of the 33rd International High Energy Physics conference, ICHEP'06 1 (2006) 421, nucl-th/0608032 .

3. Round Table Discussion I (2005): "Searching for the mixed phase of strongly interacting matter at the JINR Nuclotron", http://theor.jinr.ru/meetings/2005/roundtable/

4. Round Table Discussion II (2006): "Searching for the mixed phase of strongly interacting matter at the JINR Nuclotron: Nuclotron facility development", http://theor.jinr.ru/meetings/2006/roundtable/

5. Round Table Discussion III (2008): "Searching for the mixed phase of strongly interacting matter at the Nuclotron based Ion Collider fAcility (NICA): Physics at NICA", http://theor.jinr.ru/meetings/2008/roundtable/

6. Round Table Discussion IV (2009): "Searching for the mixed phase of strongly interacting matter at the Nuclotron based Ion Collider fAcility (NICA): Physics at NICA", http://theor.jinr.ru/meetings/2009/roundtable/

7. A.N. Sissakian et al., "Design and Construction of Nuclotronbased Ion Collider fAcility (NICA)", Conceptual Design Report, (JINR, Dubna, 2008), http://nica.jinr.ru/

8. A.N. Sissakian et al., "The MultiPurpose Detector MPD (to study Heavy Ion Collisions at NICA)", Conceptual Design Report, (JINR, Dubna, 2009), http://nica.jinr.ru/
9. D. Blaschke, D. Kharzeev, A. Sissakian, A. Sorin, O. Teryaev, V. Toneev, http://theor.jinr.ru/twiki-cgi/view/NICA/WebHome 10. A.N. Sissakian, A.S. Sorin (for the NICA collaboration), "The Nuclotron-based Ion Collider fAcility (NICA) at JINR: new prospects for heavy ion collisions and spin physics", J. Phys. G: Nucl. Part. Phys. 36 (2009) 064069.

11. A.N. Sissakian, V.D. Kekelidze, A.S. Sorin (for the NICA collaboration), "The Nuclotron-Based Ion Collider fAcility at the Joint Institute for Nuclear Research", Nucl. Phys. A 827 (2009) 630 .

\footnotetext{
a e-mail: sorin@theor.jinr.ru
}

This is an Open Access article distributed under the terms of the Creative Commons Attribution-Noncommercial License 3.0, which permits unrestricted use, distribution, and reproduction in any noncommercial medium, provided the original work is properly cited 


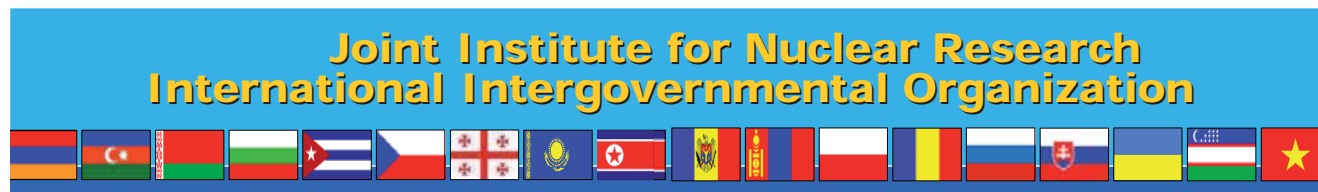

Nuclotron-based Ion Collider fAcility (NICA) at JINR: New Prospect for Heavy Ion Collisions and Spin Physics

\section{A.N.Sissakian, A.S.Sorin}

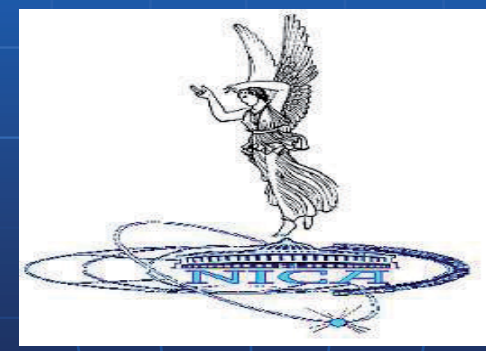

\section{Dense Matter in Heavy Ion Collisions and Astrophysics JINR, Dubna, July 172008}

JINR is an international research organization established in accordance with the intergovernmental agreement signed in 1956. At the present time, eighteen countries are the JINR Member States. JINR has an extensive and fruitful collaboration with Germany, Hungary, Italy, Serbia, and the Republic of South Africa having an Observer status at JINR.

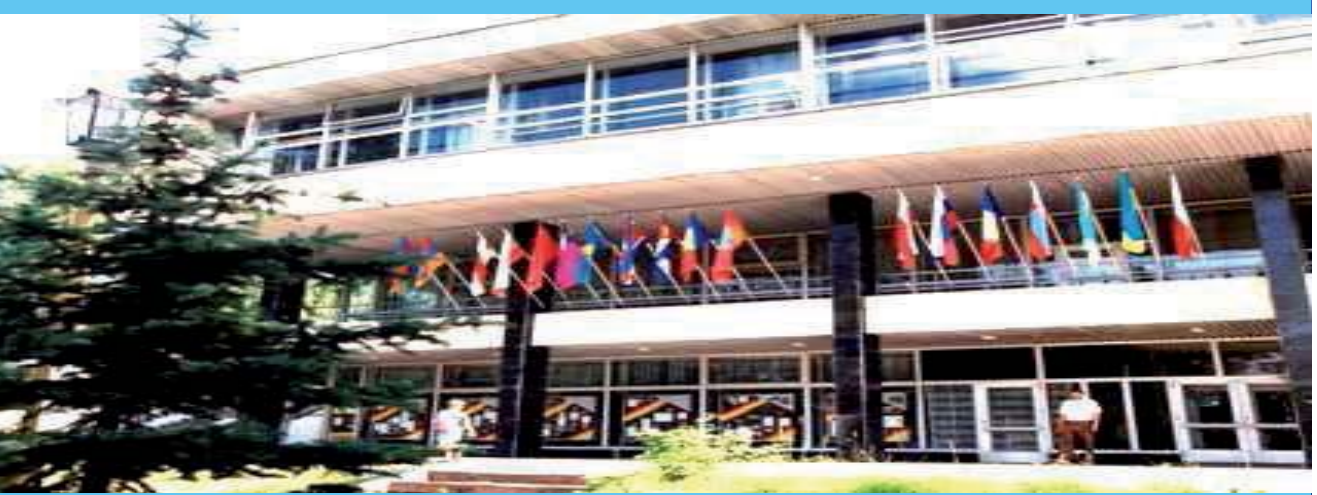

-After 52 years of scientific activity the Institute plays a world leading role in a number of branches of fundamental physics.

-Long-term scientific cooperation is established with CERN, Germany (GSI, DESY), France (GANIL, IN2P3), USA (FNAL, BNL, TJNL, LBL. LNL), Switzerland (PSI), Japan (RIKEN, KEK) and many other countries.

-The largest number of collaborative investigations are carried out with Russian research centers: IHEP (Protvino), INR RAS (Troitsk), ITEP (Moscow), Lebedev PI RAS (Moscow), BINP (Novosibirsk), Russian Research Center "Kurchatov Institute", SINP MSU (Moscow), PNPI RAS (Gatchina) and others. 


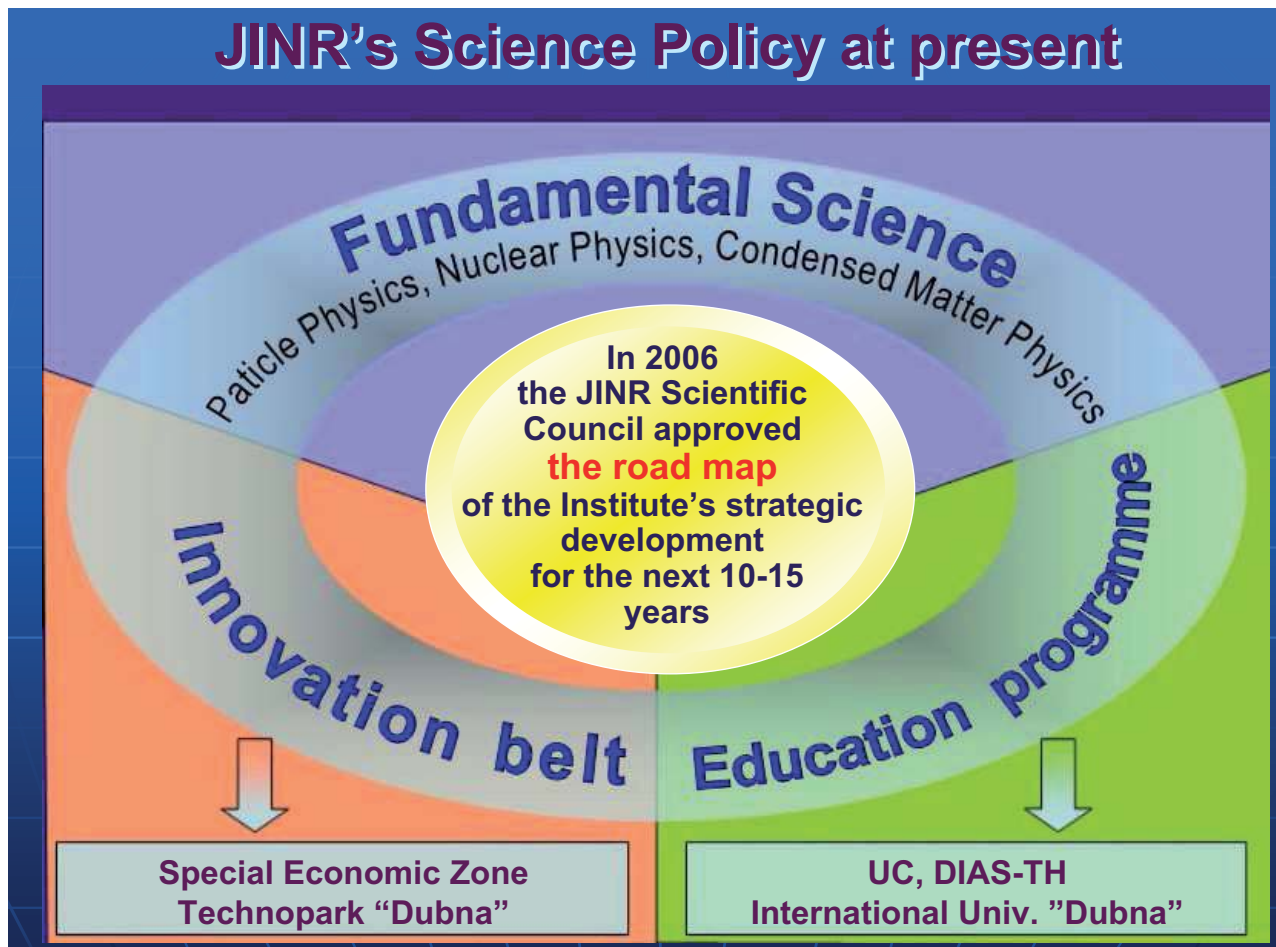

\section{JINR's research niche offered by home facilities}

$\square$ Heavy-lon Physics:

- at high energies (up to $5 \mathrm{GeV} / \mathrm{n}$ ) (in future $\sqrt{s_{N N}}=9 \mathrm{GeV}$, NICA facility)

- at low and intermediate energies (5 - $100 \mathrm{MeV} / \mathrm{n})$

$\square$ Condensed Matter Physics using nuclear physics methods
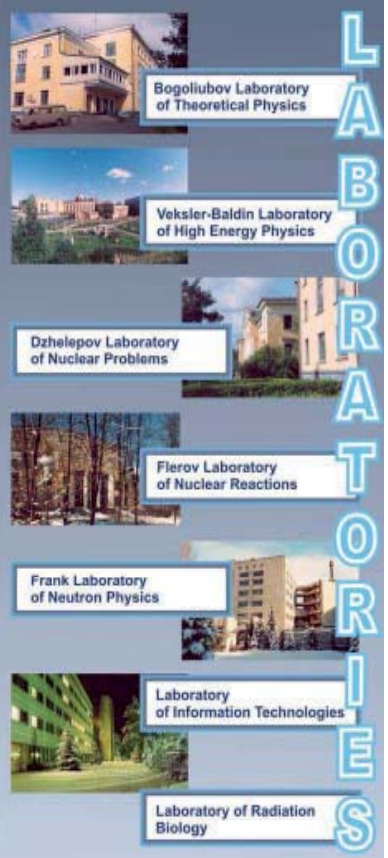
EPJ Web of Conferences

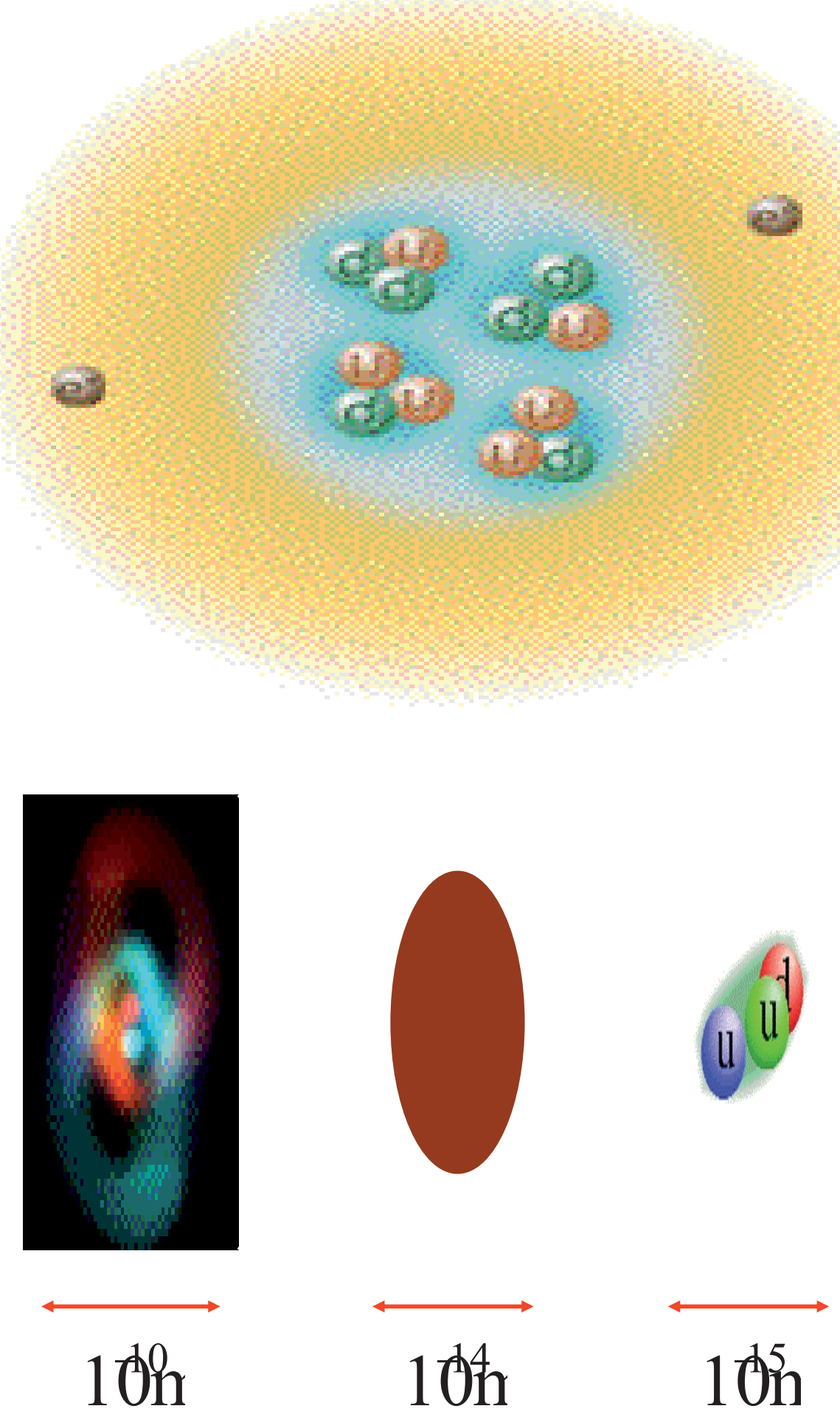


size in atoms

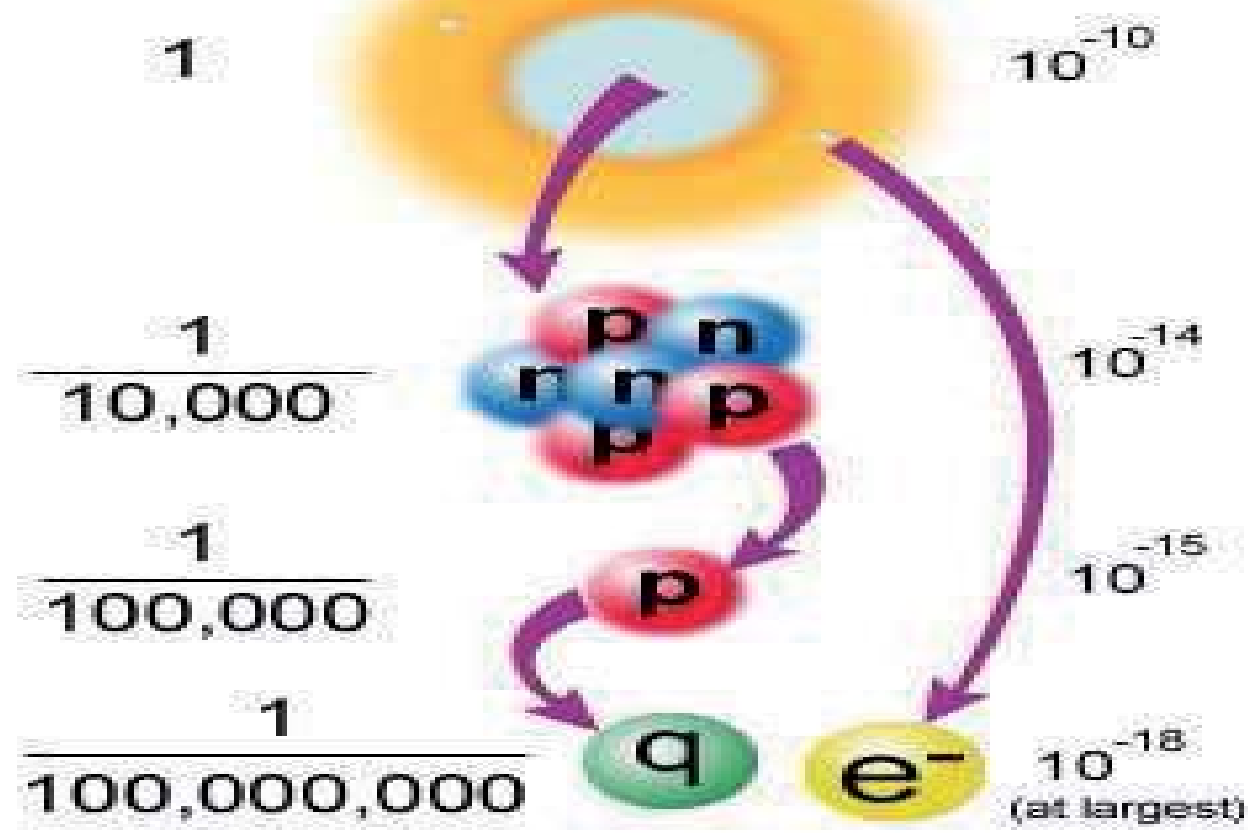

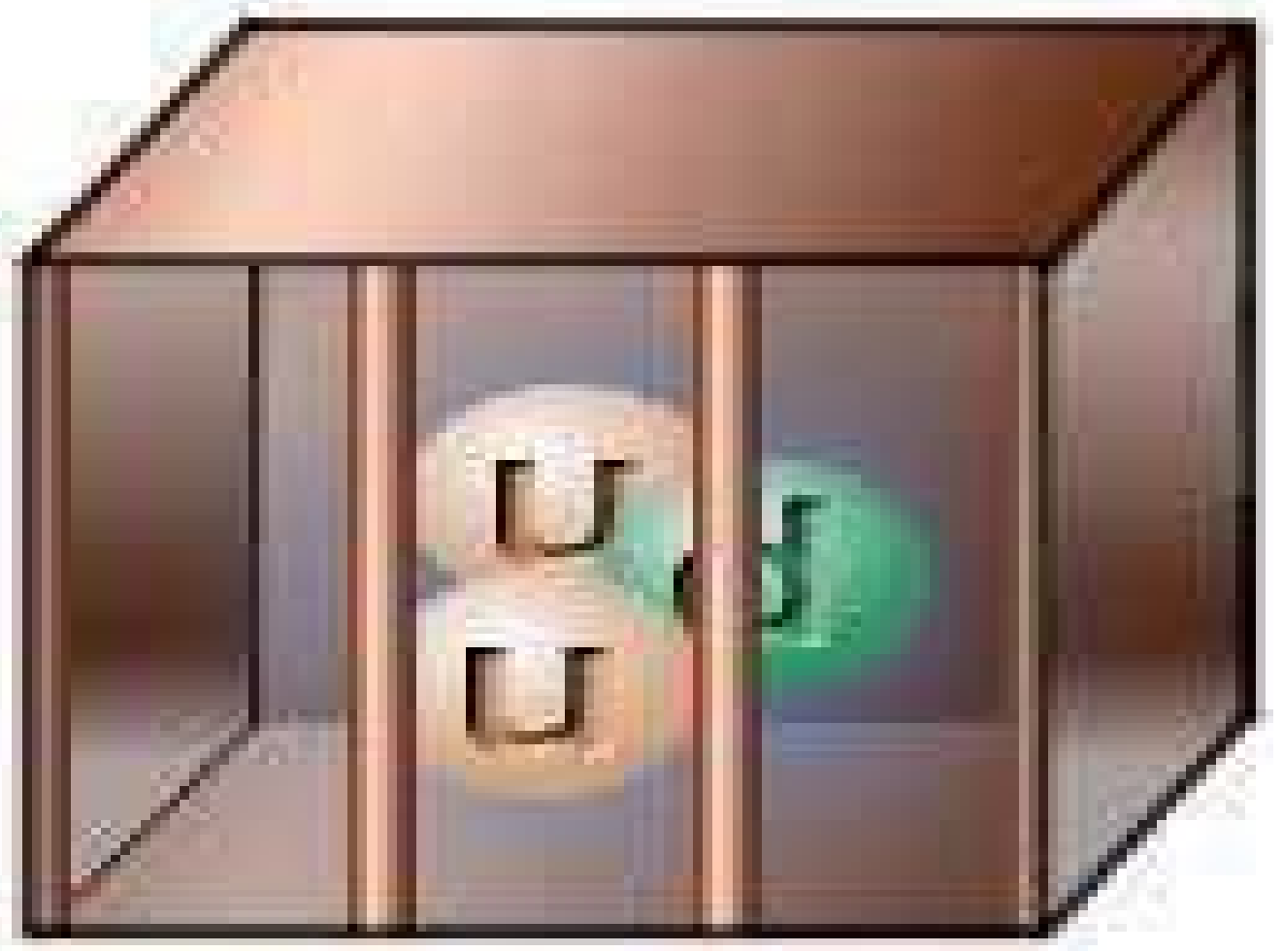


EPJ Web of Conferences

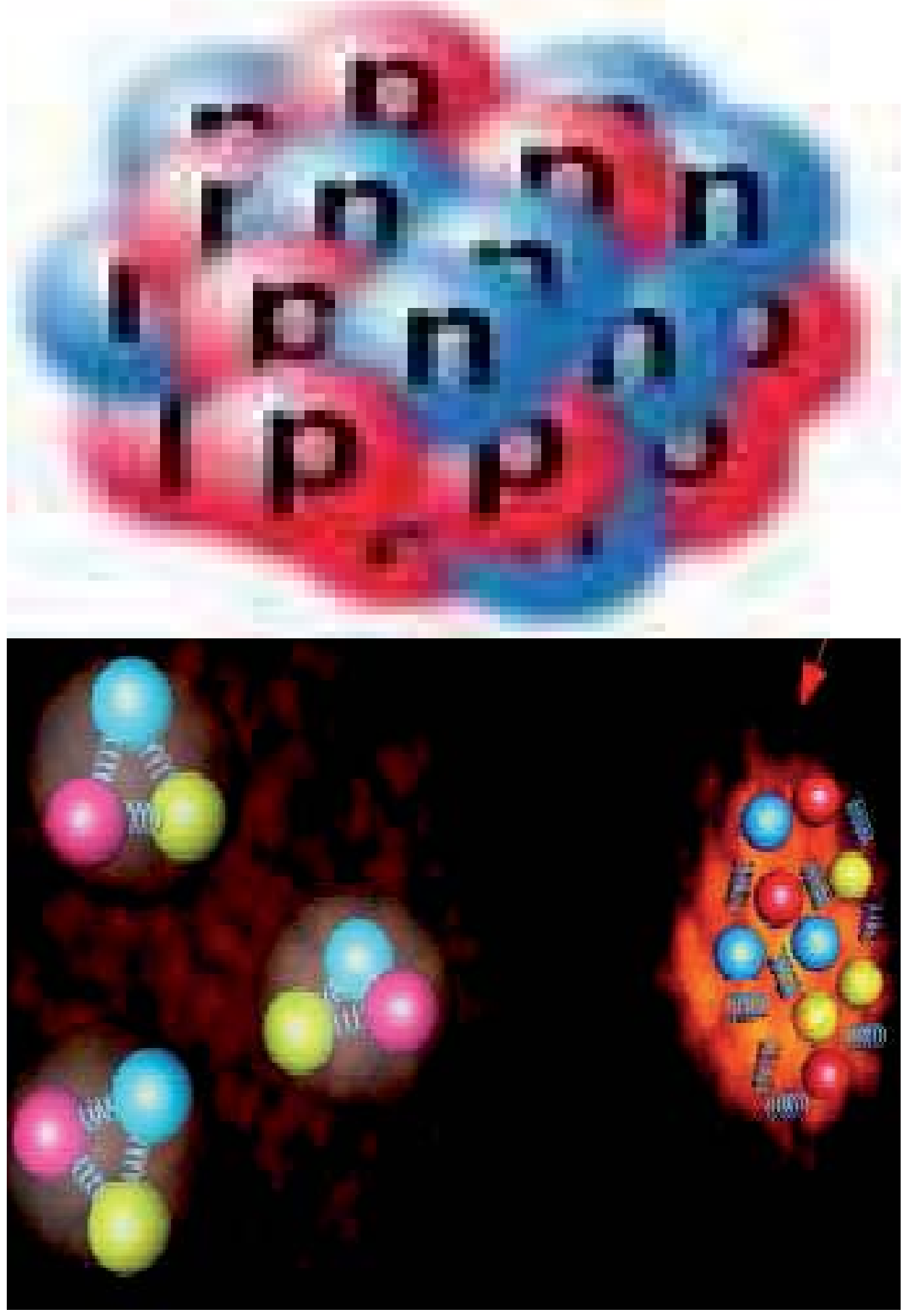



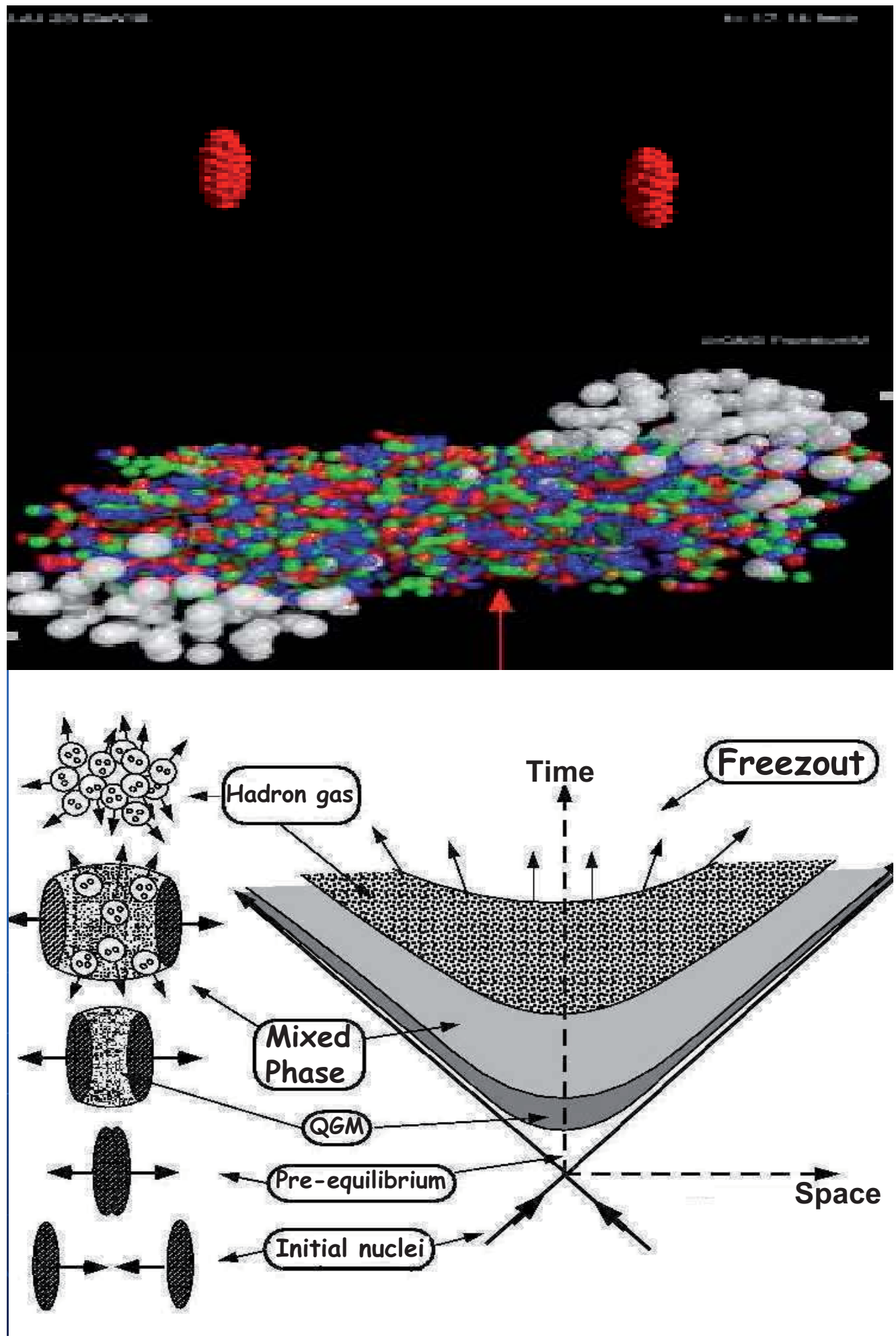

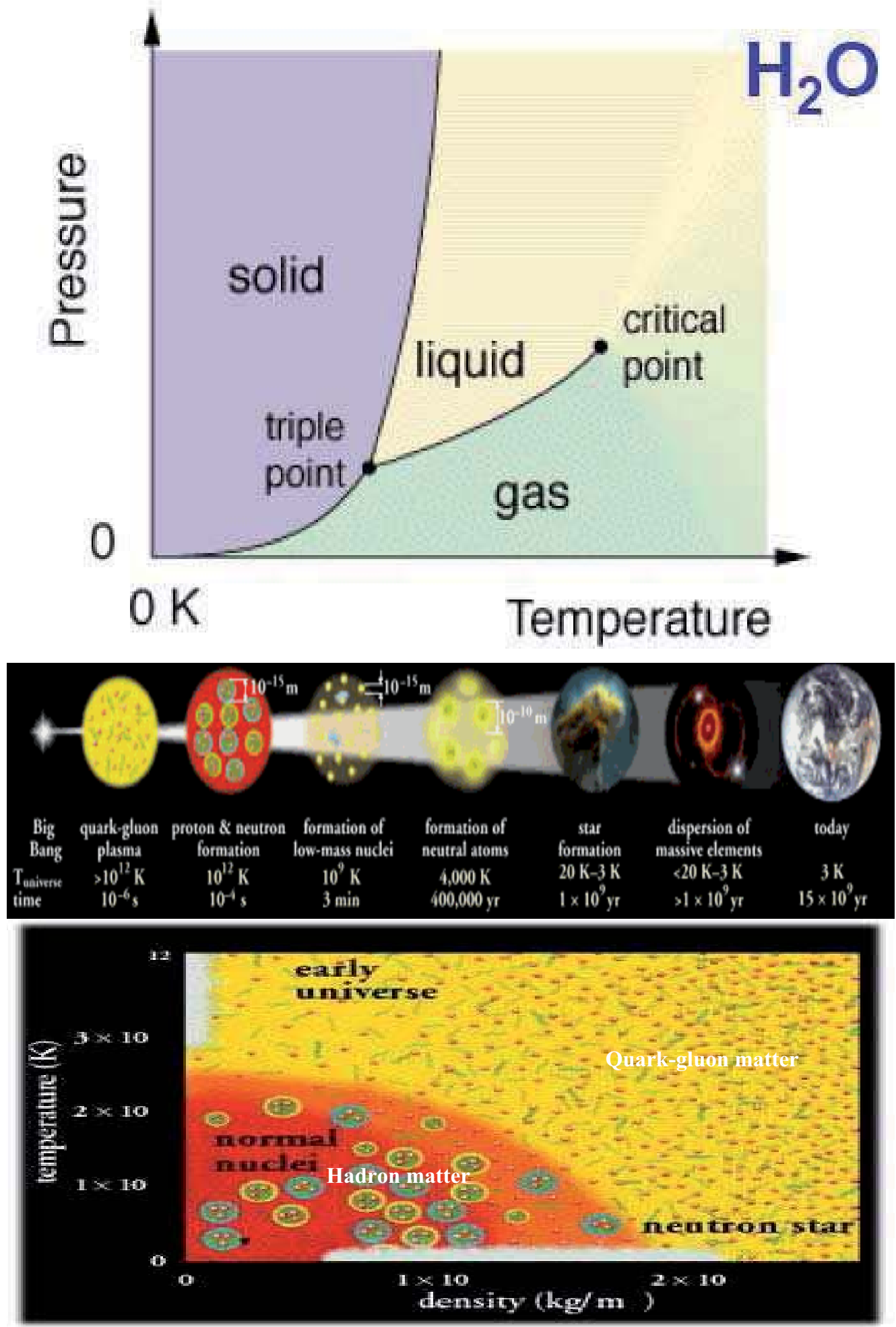


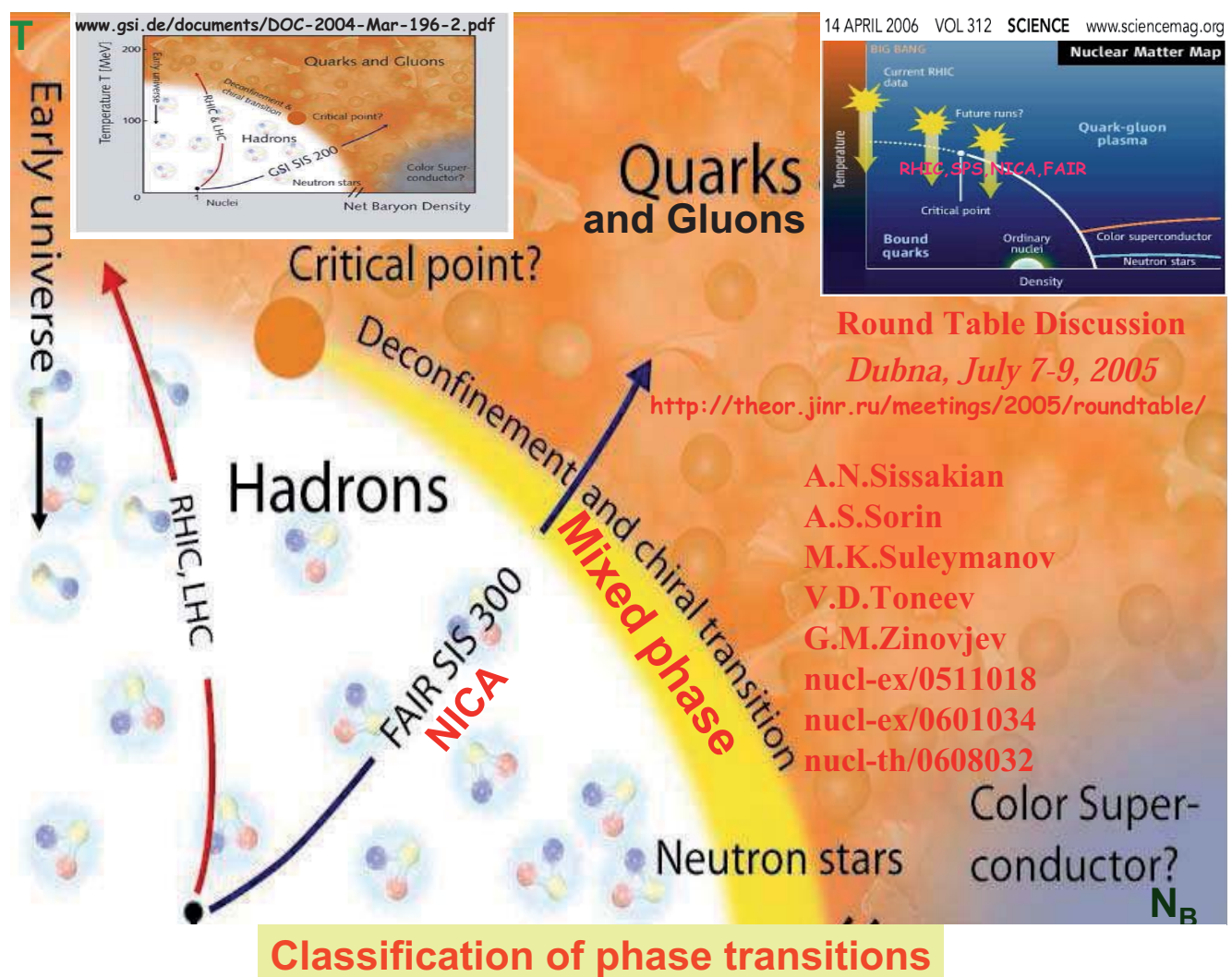

The $\underline{n}^{\text {th }}$ order phase transition:

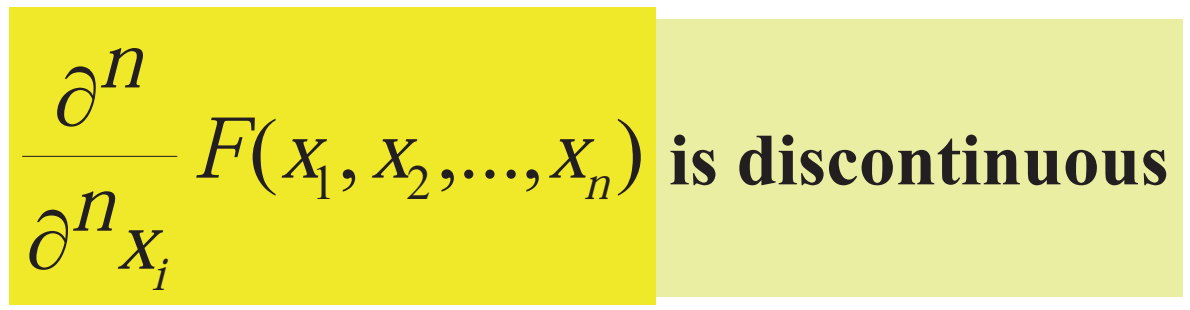

$x_{k}$ - thermodynamic variables

F - thermodynamic potential 


\section{A mixed phase in different representations}
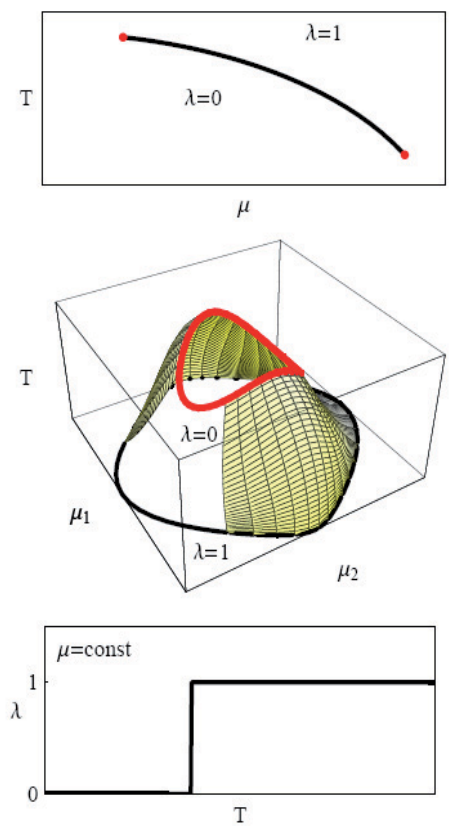

Critical end-point $=>$ critical end-line $=>$ critical end-boundary hypersurface !?

\section{Dynamical trajectories}
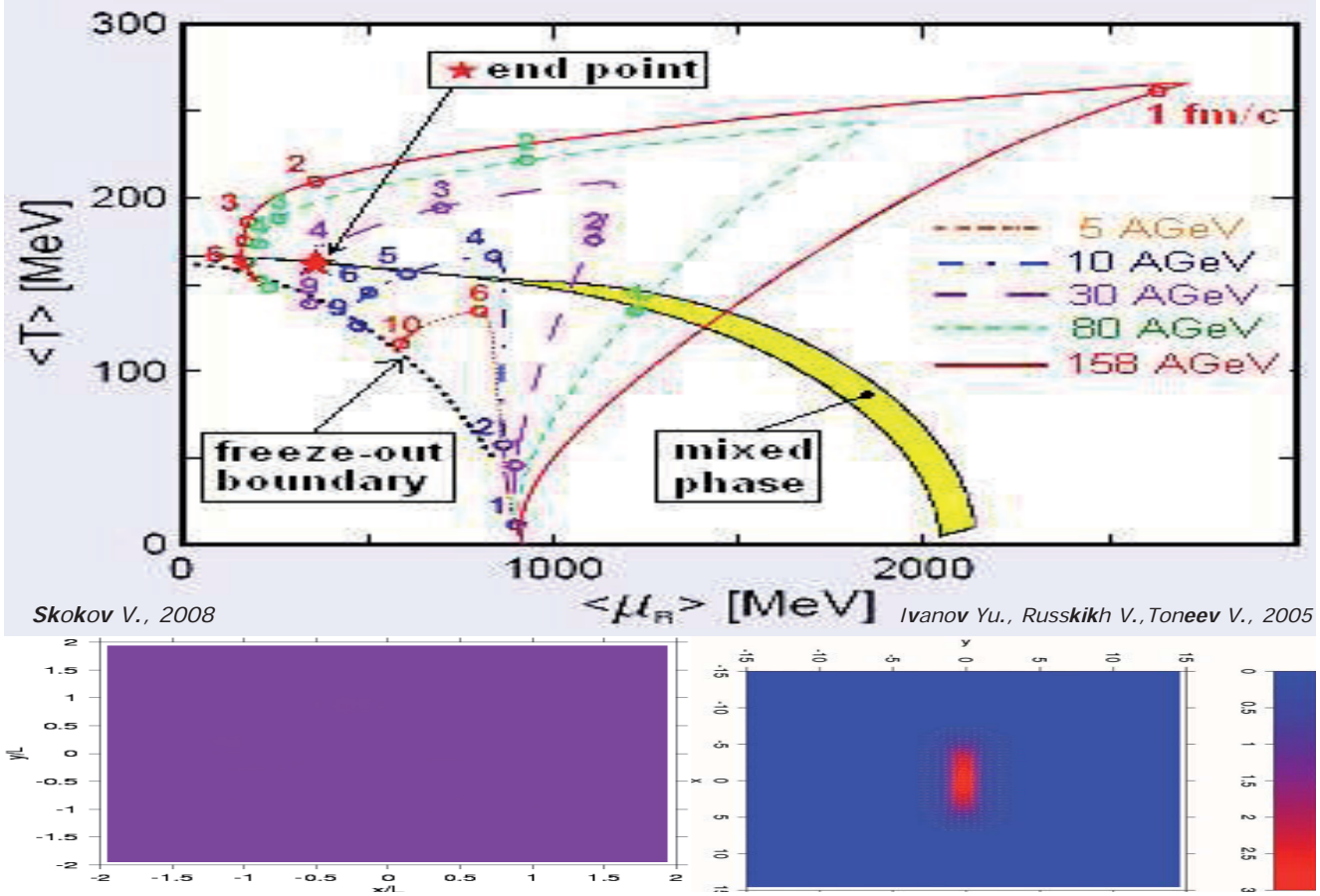


\begin{tabular}{|c|c|c|c|c|}
\hline \multicolumn{5}{|c|}{ Experimental programs } \\
\hline Facility & SPS & RHIC & NUCA & SIS-300 \\
\hline Detector & NA61 & $\begin{array}{c}\text { STAR } \\
\text { PHENIX }\end{array}$ & MPD & CBM \\
\hline Start (year) & 2010 & 2010 & $2013-2014$ & $2015-2016$ \\
\hline $\begin{array}{c}\text { Energy (for Pb-ions) } \\
\text { c.m. GeV }\end{array}$ & $4.9-17.3$ & 4.9-50 & $\leq 9$ & $\leq 8.5$ \\
\hline Physics & $C P, O D$ & CP,OD & CP,OD, HDM & CP,OD, HDM \\
\hline
\end{tabular}

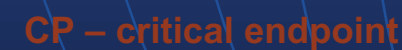

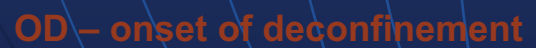
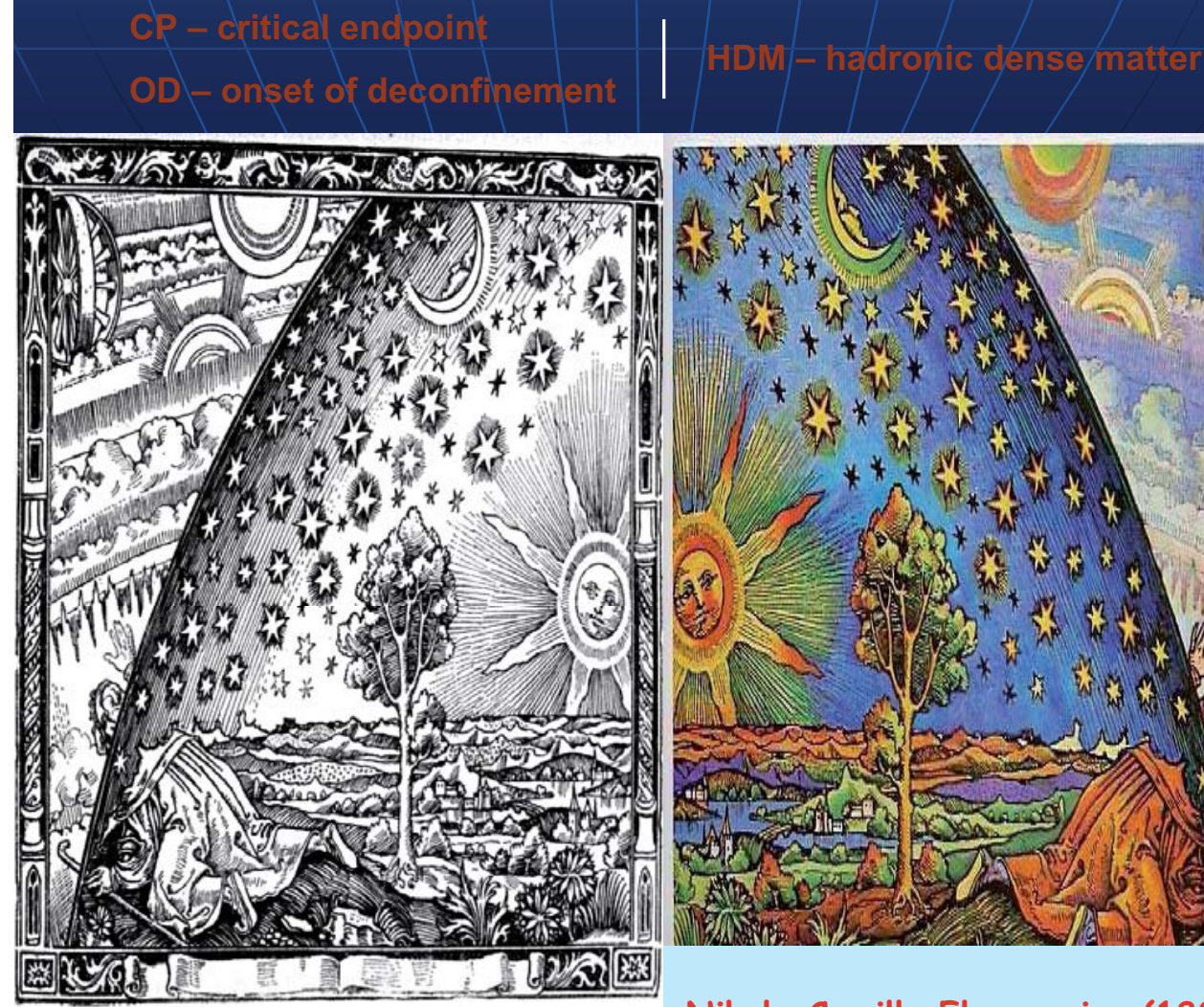

In missionnaire du mọen àge raconte quäl arait trouré le point

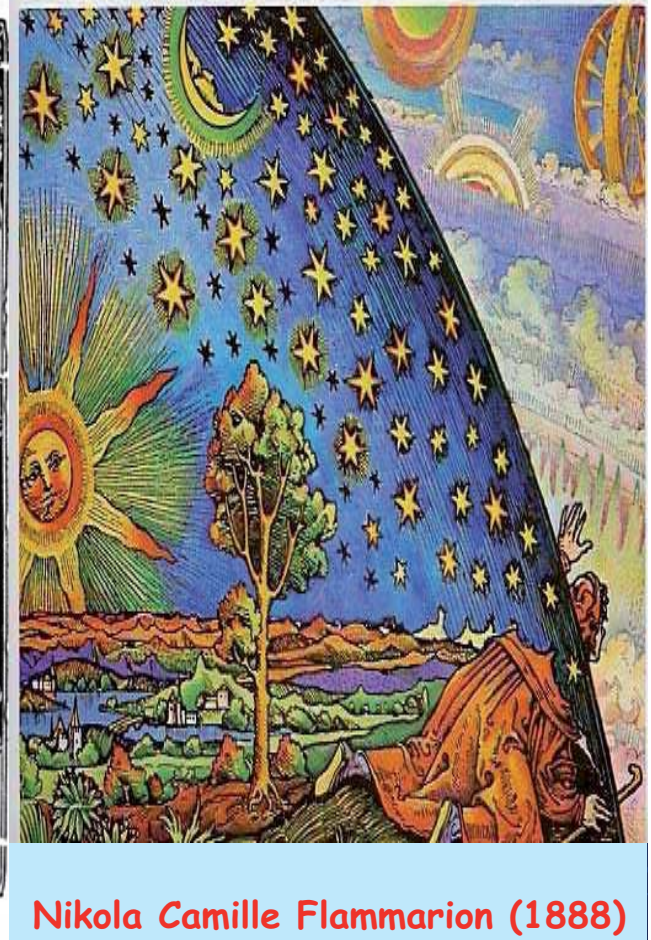
où le ciel et la Terre se touchent... 
EPJ Web of Conferences

\section{Heavy lon Accelerators}

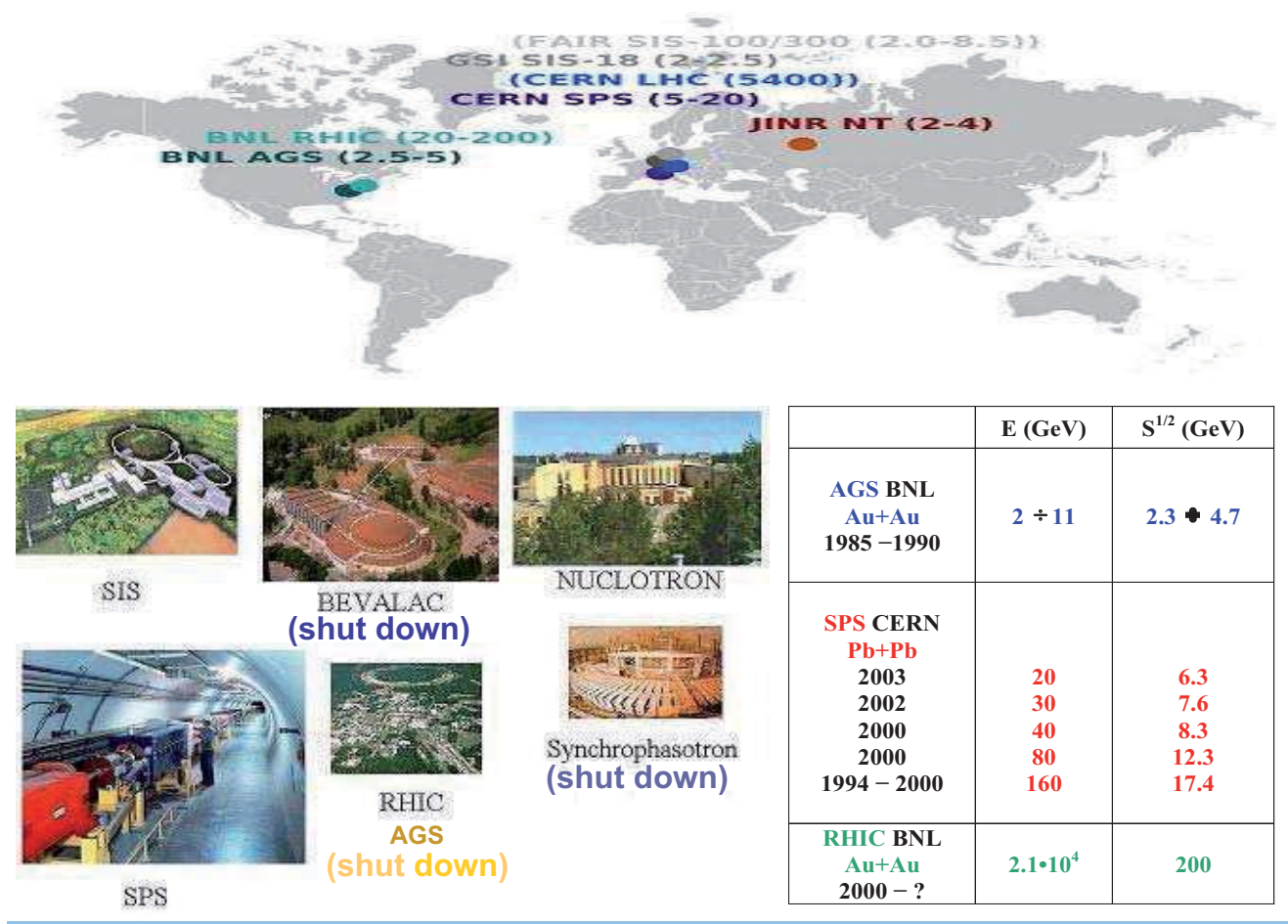

Relativistic Heary Ion Facilities

from Symchrophasotron and AGS TO NICA and FAIR

Over the last 30 years a lot of efforts have been macle to provicle the conclitions for searching for new states of strangly interacting matter uncler extreme conclitions.

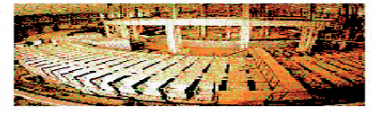

Synchrophasotron: $E_{\text {lat }} \sim 4.2$ AGeV (SWH $=390 \mathrm{~V}$

1971 - 1999 , pioneering experiments in the field of relatiwistic nuclear phvisics.

AGS: $E_{1 a b}-11$ AGeW (VSNN $\left.=5 G e V\right)$

$1986-1992$, study of compressed baryonic matter.

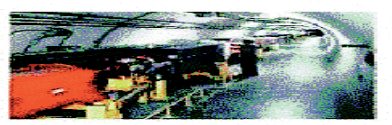

SPS: $E_{1: a b} \sim 158$ AGeV (VSNM $=18$ GeV) 1986 - up to now,

study of compressed baryonic matter.

RHIC: $\operatorname{ssm}_{\mathrm{N}}=200 \mathrm{GeV}\left(\mathrm{Elab}_{\mathrm{l}} \sim \mathrm{s0000} \mathrm{AGeV}\right)$ 1906 - uD to now.

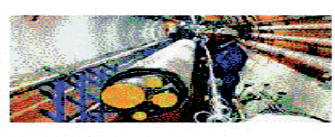

LHC:

SWN $=5600$ AGeV $\left(E_{\text {lab }} \sim 6.3 \cdot 10^{7}\right.$ AGeV $)$ 2009 - planned

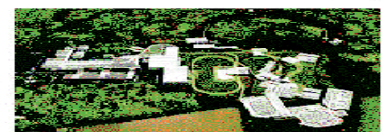

SIS 300: (FAIR GSD $E_{I a b} \sim 34$ AGeV (is 1 in $=8.5 \mathrm{GeV}$ ),

full performance will be reached in 2015 . study of compressed baryonic matter.
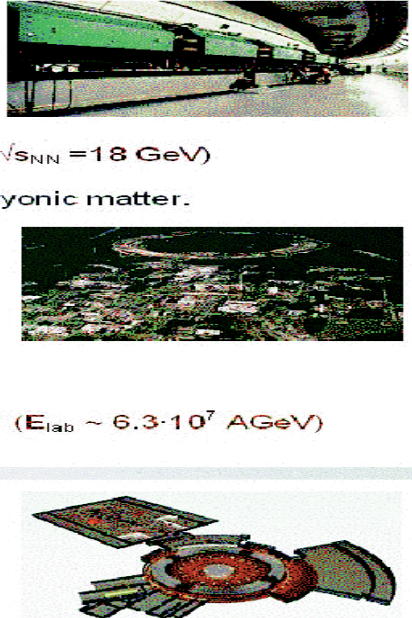

NICA: $1 S_{\text {NN }}=9$ GeV (E lab -40 AGeW). full performance will be reached in 2013 search for the mixed phase of strongly interacting matter. 


\section{BNL Brookhaven National Laboratory}

CERN European Organization for Nuclear Research

AGS (BNL) Alternative Gradient Synchrotron

SPS (CERN) Super Gradient Synchrotron

RHIC (BNL) Relativistic Heavy Ion Collider

LHC (CERN) Large Hadron Collider

JINR NUCLOTRON

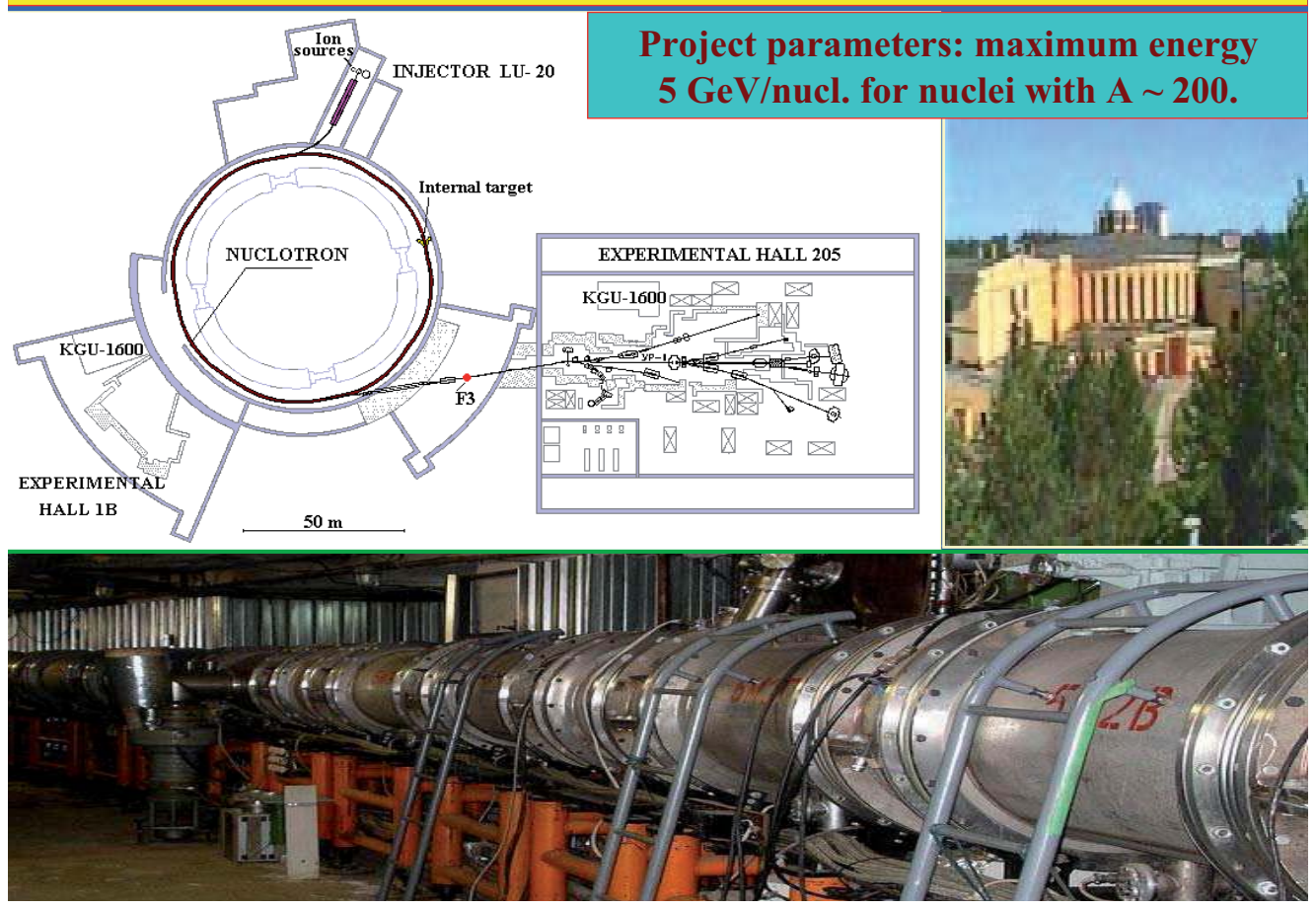




\section{The main goal of the NICA project is an experimental study of hot and dense nuclear matter and spin physics}

\section{These goals are proposed to be reached by:}

- development of the existing accelerator facility (1st stage of the

NICA accelerator programme: Nuclotron-M subproject) as a basis for generation of intense beams over atomic mass range from protons to uranium and light polarized ions;

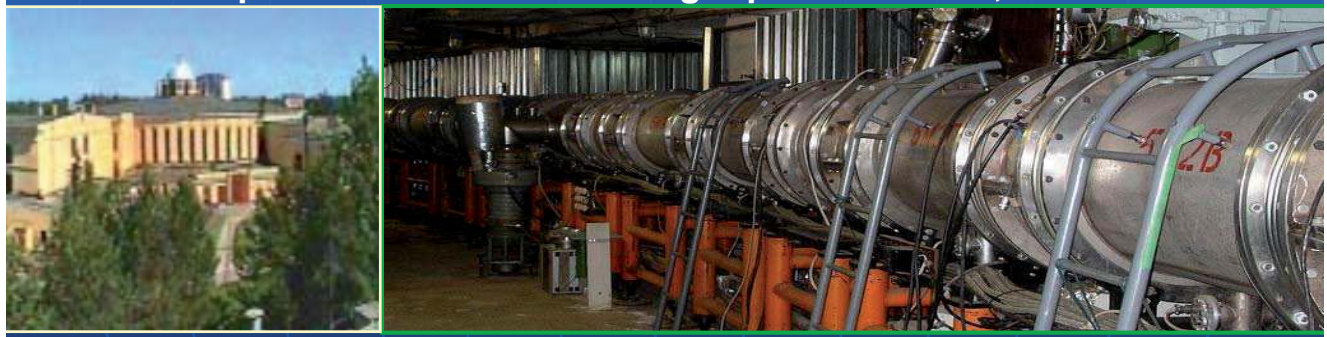

- design and construction of heavy ion collider with maximum collision energy of $\sqrt{s_{\mathrm{NN}}}=9 \mathrm{GeV}$ and average luminosity $10^{27} \mathrm{~cm}^{-2} \mathrm{~s}^{-1}$ (for $\mathrm{U}^{92+}$ ), and polarized proton beams with energy $\sqrt{s} \sim 25 \mathrm{GeV}$ and average luminosity $>10^{30} \mathrm{~cm}^{-2} \mathrm{~s}^{-1}$;

- design and construction of the MultiPurpose Detector (MPD).

\section{Scheme of the NICA compex}

Injector: $2 \times 10^{9}$ ions/pulse of ${ }^{238} \mathrm{U}^{32+}$ at energy $6 \mathrm{MeV} / \mathrm{u}$

Storage of

15 bunches $\times 1.10^{9}$ ions per ring at $3.5 \mathrm{GeV} / \mathrm{u}$,

electron and/or stochastic cooling

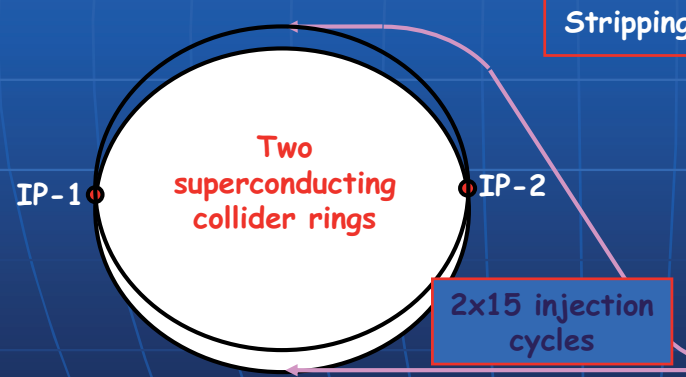

2(3?) single-turn injections, storage of $3.2 \times 10^{9}$. acceleration up to $50 \mathrm{MeV} / \mathrm{u}$, electron cooling. acceleration up to $400 \mathrm{MeV} / \mathrm{u}$ g $(40 \%)^{238} U^{32+} \Rightarrow$

Nuclotron (45 Tm) injection of one bunch of $1.1 \times 10^{9}$ ions, acceleration up to $3.5 \mathrm{GeV} / \mathrm{u} \max$. 


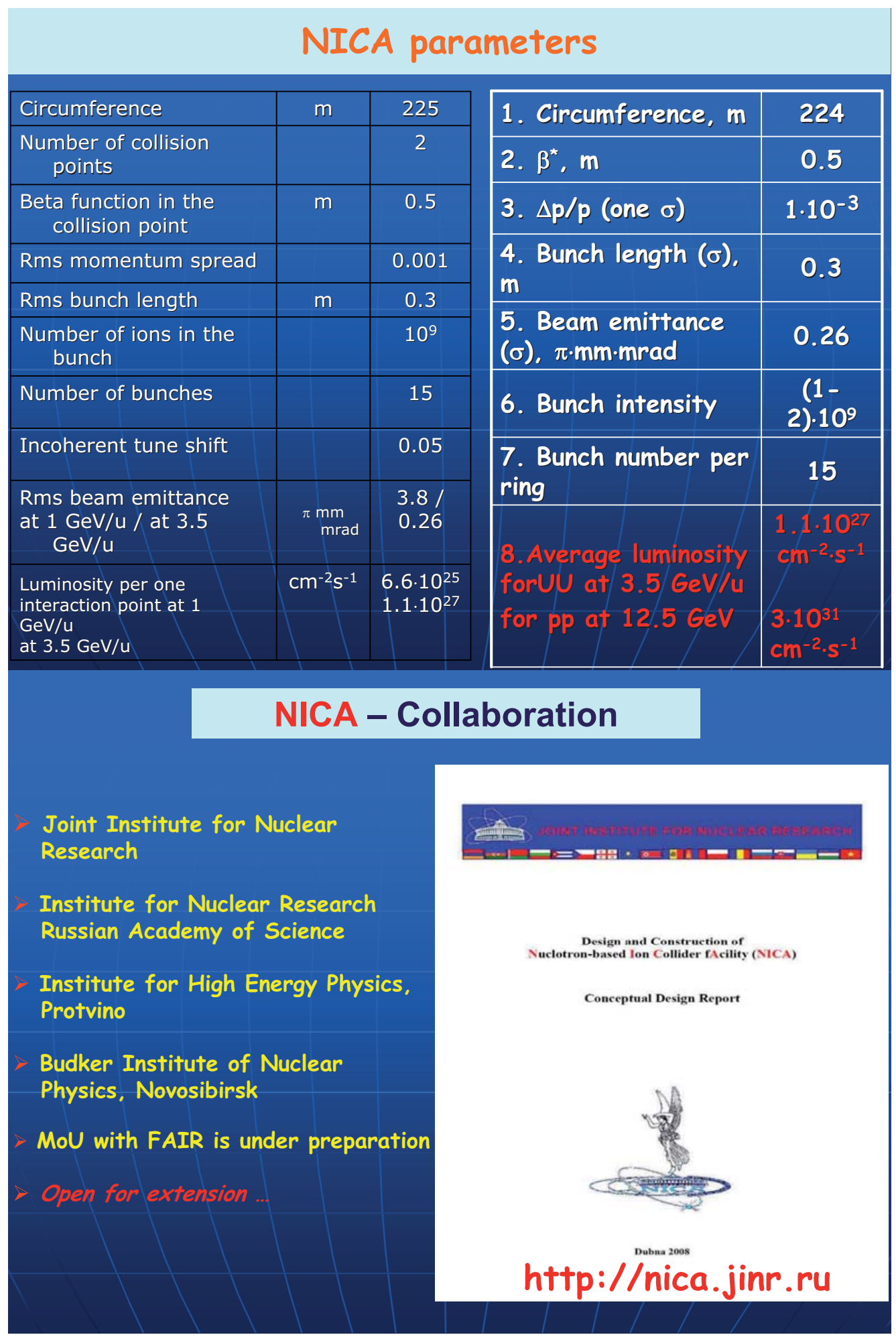


- Stage 1: years $2007-2009$

- Upgrate and Development of the Nuclotron facility

- Preparation of Technical Design Report of the NICA and MPD

- Start prototyping of the MPD and NICA elements

- Stage 2: years $2008-2012$

Design and Construction of NICA and MPD

- Stage 3: years $2010-2013$

- Assembling

- Stage 4: year 2013 - 2014

- Commissioning

NICA provides unique possibility for the heavy ion physics program:

1. Heavy ion beams in wide energy range:

$$
\sqrt{s}=4-9 A \mathrm{GeV}
$$

2. Possibility to perform atomic mass and centrality scan

3. Few intersection points for detectors with large energy-independent acceptance

4. High luminosity $L \sim 10^{27} \mathrm{CM}^{-2} \mathrm{c}^{-1}$ 


\section{High baryonic densities}

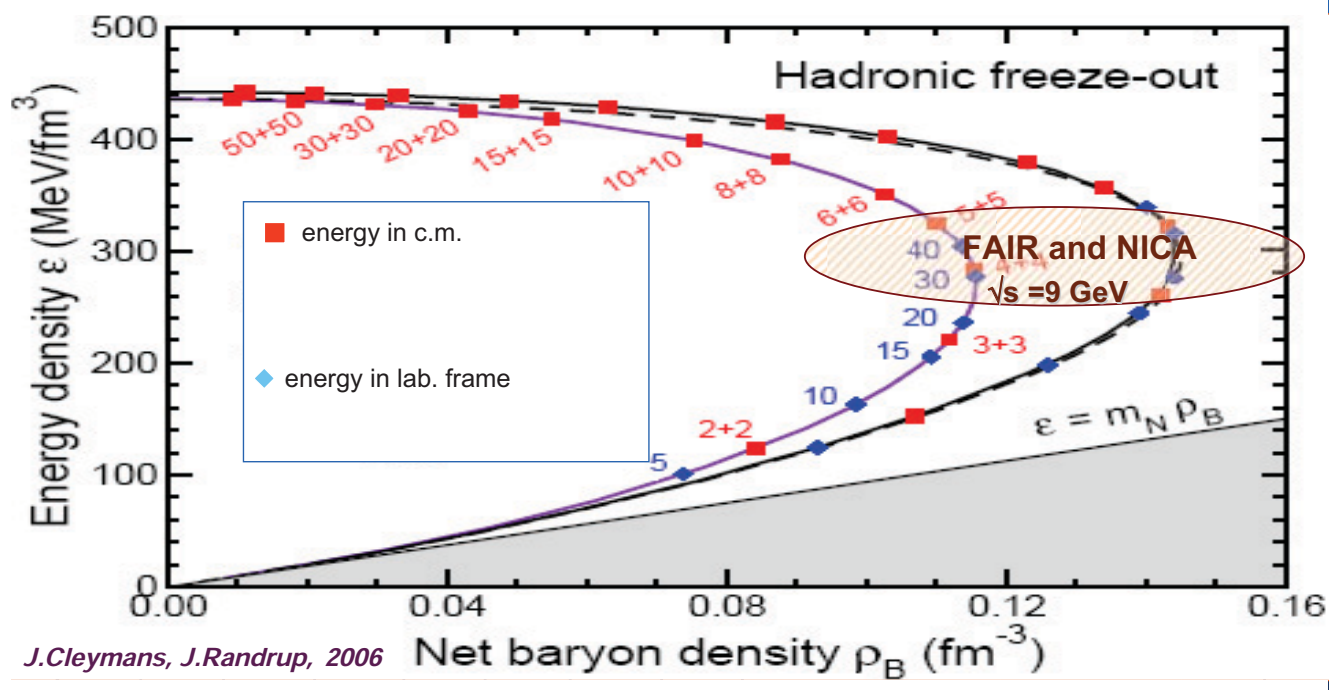

Maximal baryonic densities on freeze-out curve! $\Rightarrow$ High densities on interaction stage!?

\section{Critical points}

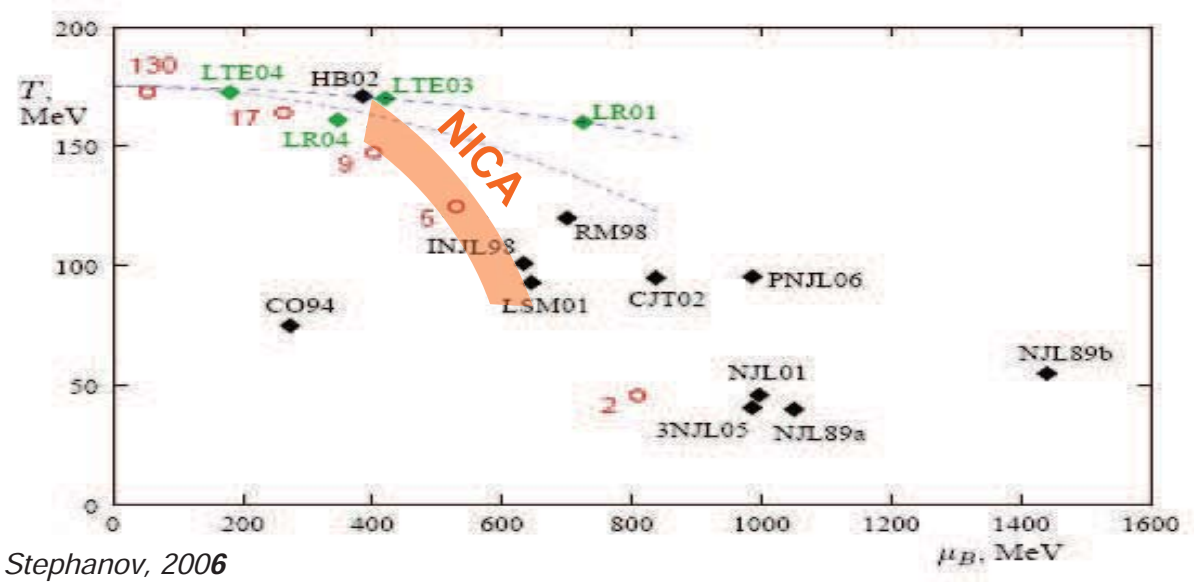

M. Stephanov, 2006

Lattice QCD

- Chemical freeze-out for different energies $\sqrt{s}$ 


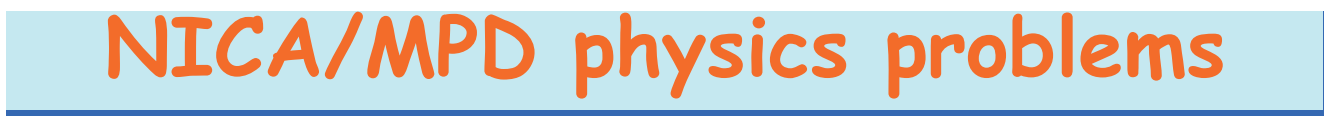

Study of in-medium properties of hadrons and nuclear matter equation of state including a search for possible signs of deconfinement and/or chiral symmetry restoration phase transitions and

QCD critical endpoint

\section{Experimental observables:}

Scanning in beam energy and centrality of excitation functions for

* Multiplicity and global characteristics of identified hadrons including multi-strange particles

2 Fluctuations in multiplicity and transverse momenta

* Directed and elliptic flows for various indentified hadrons

* particle correlations

\& Dileptons and photons

\section{What to measure}

Multistrange hyperons. The yields, spectra and collective flows of (multi) strange hyperons are expected to provide information on the early and dense phase of the collision. Therefore, these particles are promising probes of the nuclear matter equation-of-state at high baryon density.

Event-by-event fluctuations. The hadron yields and their momenta should be analyzed eventwise in order to search for nonstatistical fluctuations which are predicted to occur in the vicinity of the critical endpoint and when penetrating the coexistence phase of the first order

deconfinement phase transition.

\&BT correlations. Measurement of short range correlations between hadrons $\pi, K, p, \wedge$ allows one to estimate the space-time size of a system formed in nucleus-nucleus interactions. Along with the increase of fluctuations, the spatial size of the system is expected to be getting smaller near the deconfinement phase transition due to softening of the equation of state (the "softest point" effect).

s. Penetrating probes. Measurements of dilepton pairs permit to investigate the in-medium spectral functions of low-mass vector mesons which are expected to be modified due to effects of chiral symmetry restoration in dense and hot matter. Specific properties of the $\boldsymbol{\sigma}$ meson, the order parameter of chiral symmetry restoration, may be in principle detected near the phase boundary via particular channel of $\sigma$-decay into dileptons or correlated $\gamma \gamma$-pairs. 


\section{HBT identical particle correlations}

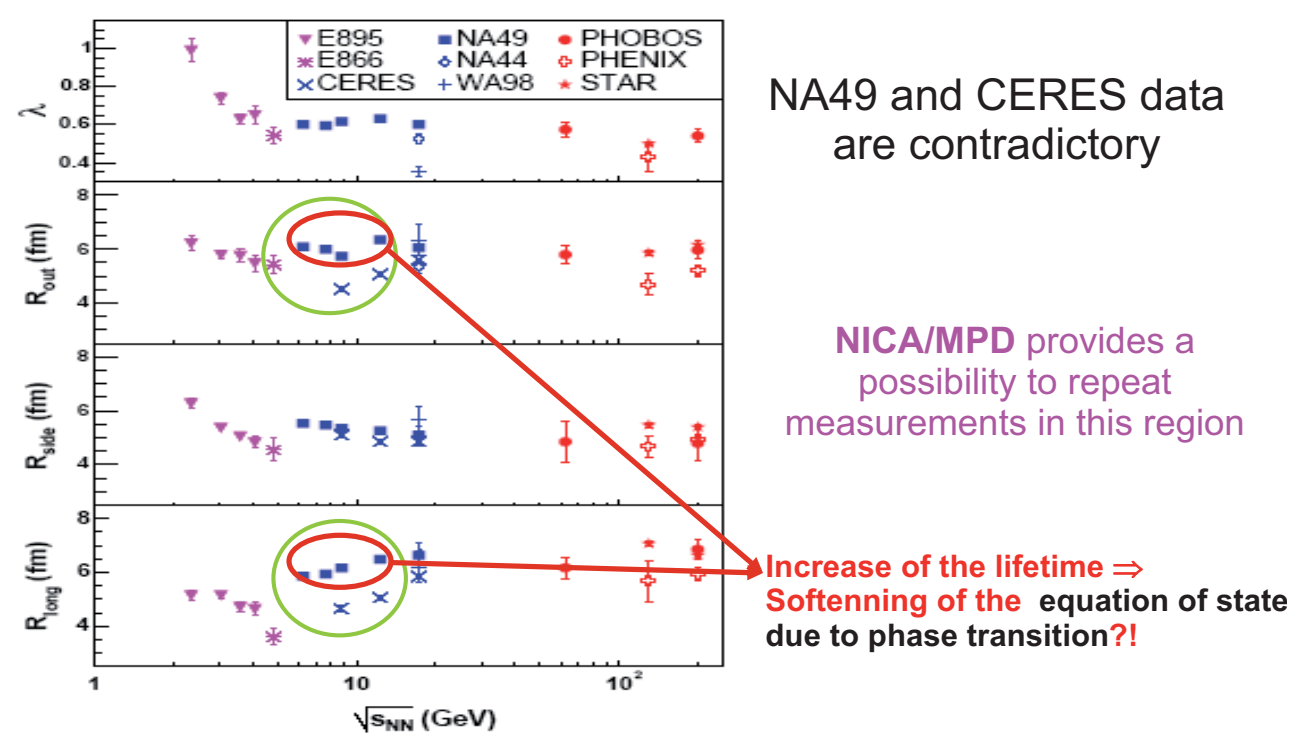

B.Back et al., 2006

\section{Excitation functions of particle ratios}

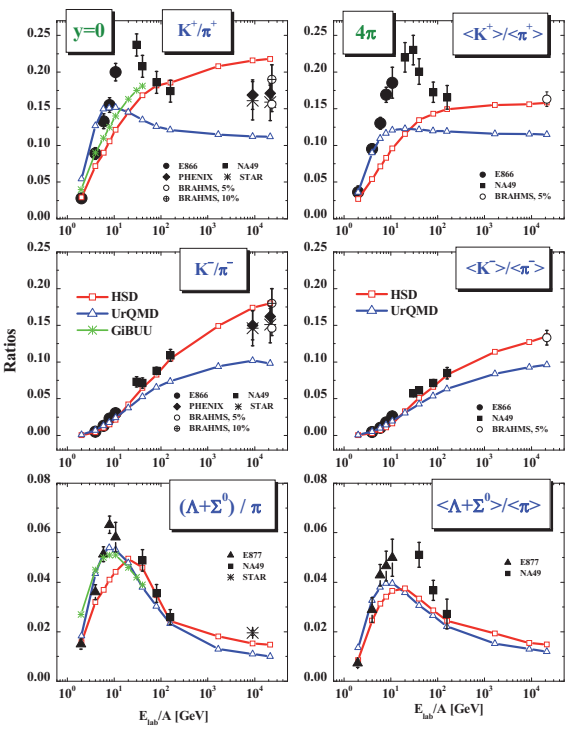

\section{HSD, UrQMD, GiBUU}

Experimental data:

E896, NA49, STAR, PHENIX, BRAHMS

Exp. data (particularly a maximum at E 30 AGeV are not well reproduced within the hadron-string picture $=>$ evidence for nonhadronic degrees of freedom 


\section{Inverse slopes of transverse mass spectra}
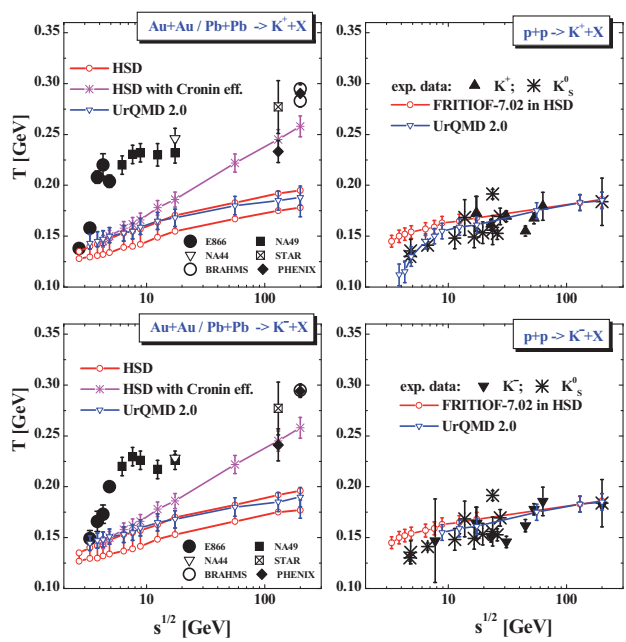

E.Bratkovskaya et al.,(2004) $d N / m_{T} d m_{T} \sim \exp \left(-m_{T} / T\right)$

Hadronic rescattering has only a small effect on the kaon slope in UrQMD and HSD models

The hadron-string picture fails? => New degrees of freedom are missing (collectivity?)

The beginning of flattening correlates with the position of maximum in $\mathrm{K}^{+} / \pi^{+}$,

E 20-30 AGeV

\section{Directed $v_{1}$ and elliptic $v_{2}$ flows}

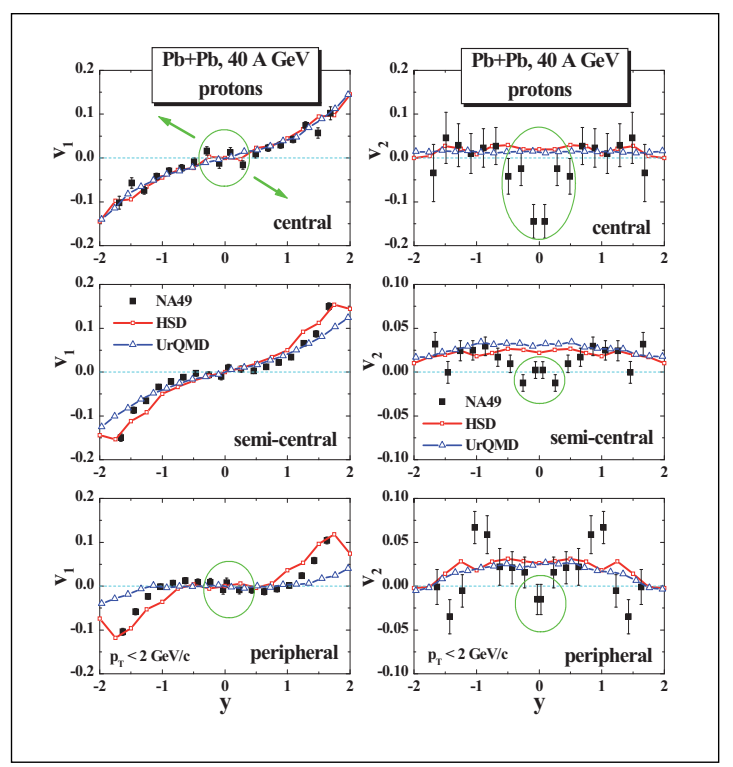

H.Stoecker et al., 2005 


\section{Fluctuations: theoretical status}

Lattice QCD predictions: Fluctuations of the

clessitity (susceptibility) at $\mu_{-} B>0 \quad$ C.Allton et al., 2003)
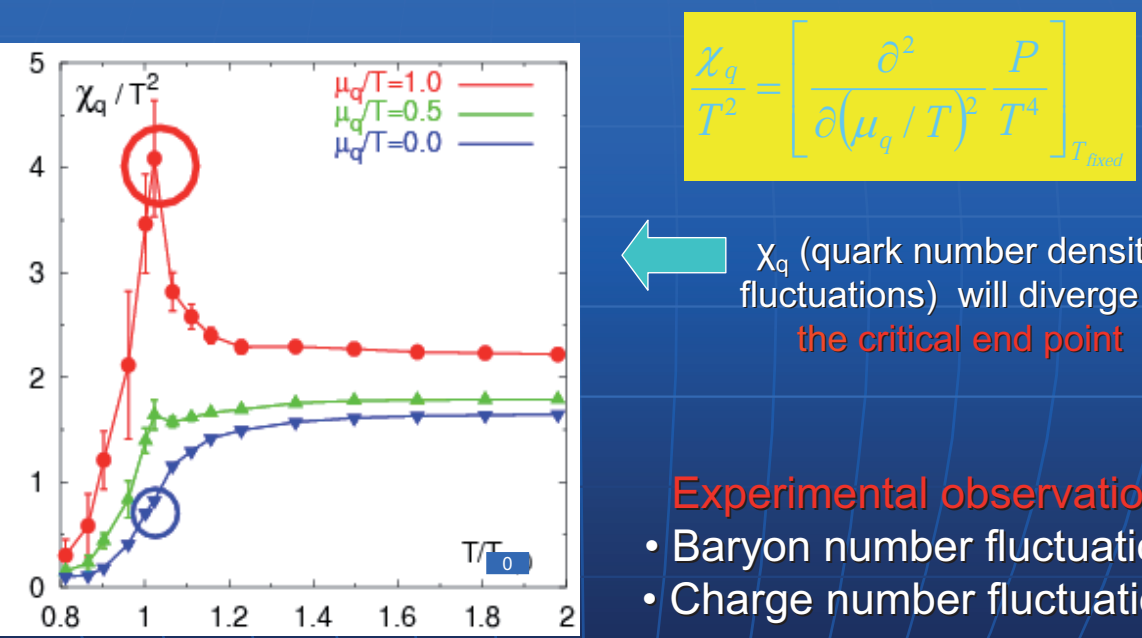

$x_{q}$ (quark number density fluctuations) will diverge at

\section{Experimental observation:}

- Baryon number fluctuations

- Charge number fluctuations

\section{Collective flows}

Interactions between constituents lead to a pressure gradients $\Rightarrow>$ spatial asymmetry is converted in asymmetry in momentum space$$
\Rightarrow \text { collective flows }
$$

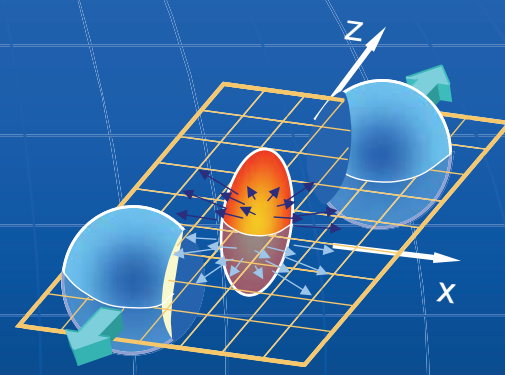

$\frac{d N}{d y p_{T} d p_{T} d \varphi}=\frac{d N}{d y p_{T} d p_{T}} \frac{1}{2 \pi}\left(1+2 v_{1} \cos (\varphi)+2 v_{2} \cos (2 \varphi)+\ldots\right)$

directed

flow elliptic flow 


\section{Correlation femtoscopy of identical particles}

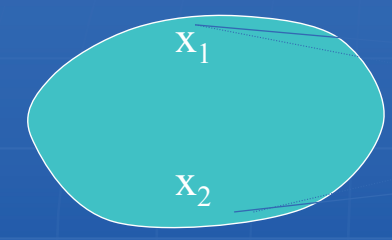

$\mathrm{p}_{2} \mathrm{p}_{1}$

$$
q=p_{1}-p_{2}, \Delta x=x_{1}-x_{2}
$$

$$
\begin{aligned}
C_{2}=1+(-1)^{S}<\operatorname{Cos} q \Delta x>\rightarrow 1 & +\lambda \exp \left(-R_{\text {long }}^{2} q_{\text {long }}^{2}\right. \\
& -R_{\text {side }}^{2} q_{\text {side }}^{2}-R_{\text {out }}^{2} q_{\text {out }}^{2} \\
& \left.-2 R_{\text {out }}^{2} q_{\text {out }} q_{\text {long }}\right)
\end{aligned}
$$




\section{Signals of chiral symmetry restoration}

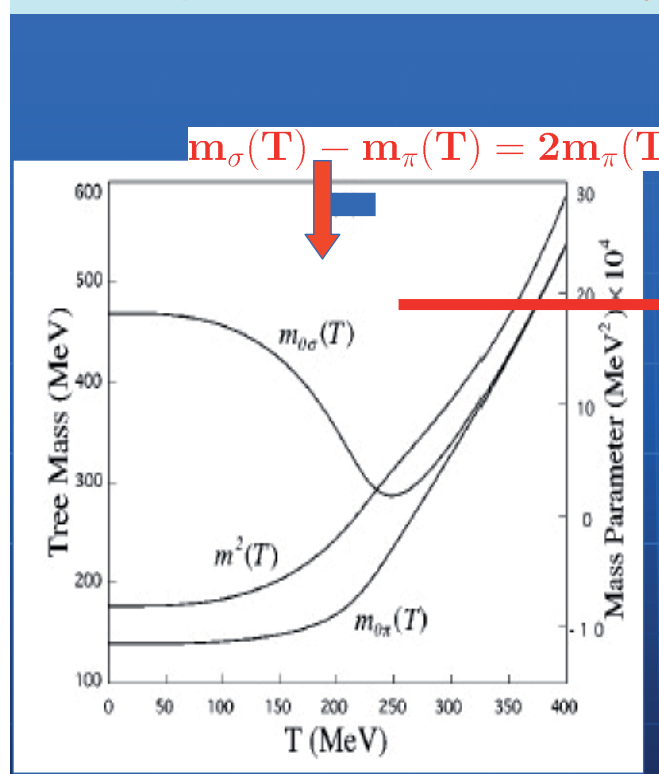

S.Chiki, T.Hatsuda, 1998
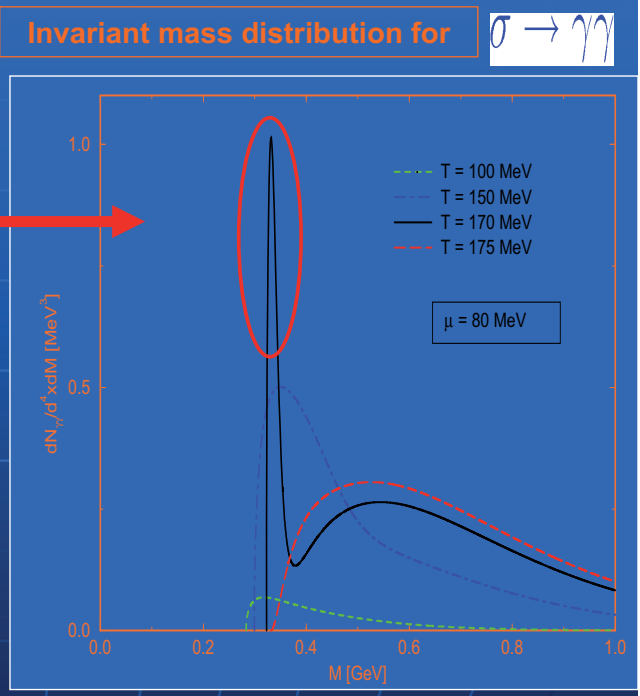

M.Volkov et al., 1998

\section{MPD conceptual design}

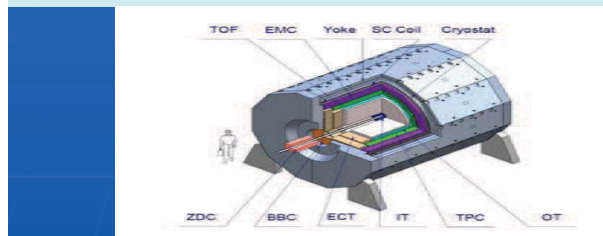

WPD basic geometry

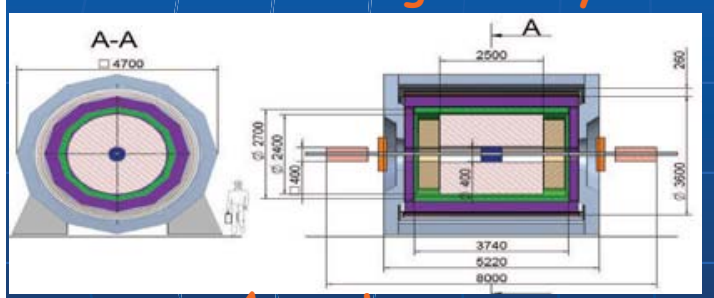

Acceptance

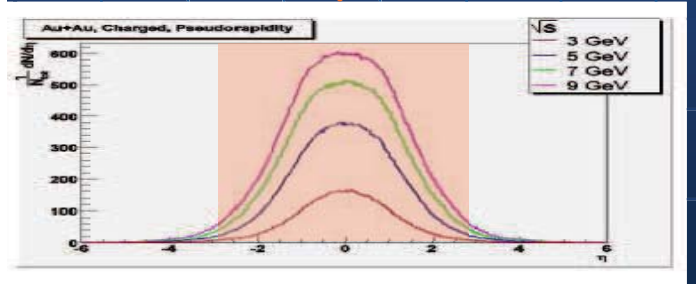

Inner Tracker (IT) silicon strip detector / micromegas for tracking close to the interaction region.

Barrel Tracker (BT) - TPC + Straw (for tagging) for tracking \& precise momentum measurement in the region $-1<\eta<1$

End Cap Tracker (ECT) - Straw (radial) for tracking \& p-measurement at $|\eta|>1$

Time of Flight (TOF) - RPC (+ start/stop sys.) to measure Time of Flight for charged particle identification.

Electromagnetic Calorimeter (EMC) for $\pi^{0}$ reconstruction \& electron/positron identification.

Beam-Beam Counters (BBC) to define centrality (\& interaction point).

Zero Degree Calorimeter (ZDC) for centrality definition. 


\section{MPD - Collaboration}

Joint Institute for Nuclear Research

Institute for Nuclear Research Russian Academy of Science

Bogolyubov Institute of

Theoretical Physics, NASUk

Skobeltsyn Institute of Nuclear

Physics of Lomonosov MSU, RF

Institute of Apllied Physics, Academy of Science Moldova

Open for extension

A consortium involving GSI, JINR \& other centers for IT module development \& production is at the organizational stage

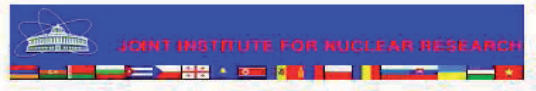

\section{Versioa 1}

The MultiPurpose Detector (MPD) to study Heavy Ion Collisions at NICA

Letter of Intent

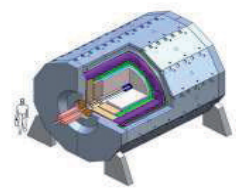

Dubna, 2008

http://nica.jinr.ru

\section{On the Spin Program at NICA}
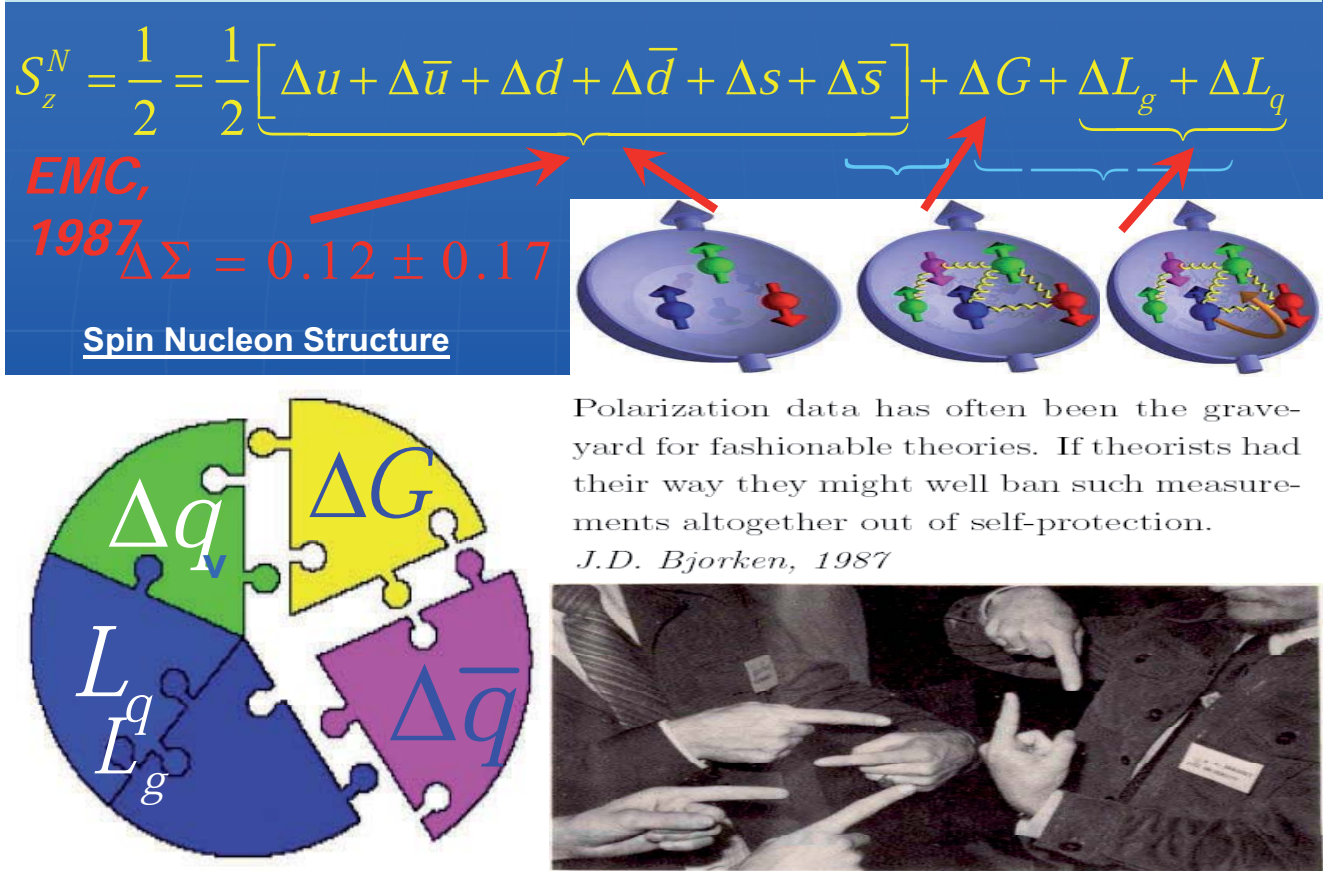

Polarization data has often been the graveyard for fashionable theories. If theorists had their way they might well ban such measurements altogether out of self-protection. J.D. Bjorken, 1987

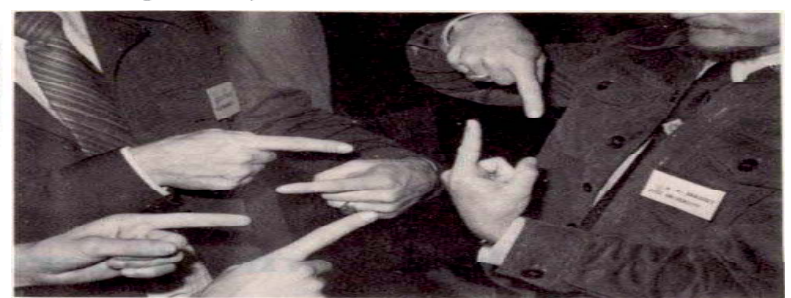




\section{NICA could provide unique possibilities for the spin program :}

1. Accelerate at NUCLOTRON polarized high energy proton and deuterons

2. Have high luminosity ( $>10{ }^{30} \mathrm{CM}^{-2} \mathrm{c}^{-1}$ )

3. Have high polarization $(>50 \%$ )

4. Rotate spin L/T

5. Polarization measure $\sim 3 \%$

6. Construct adequate ( $4 \pi$ geometry) detector

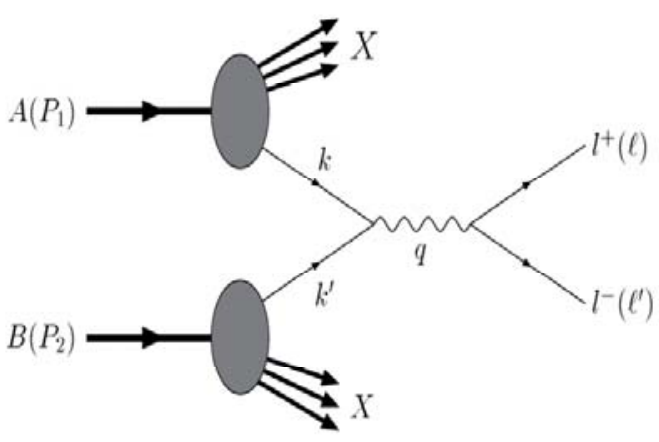

\section{Spin program}

Studies of Drell-Yan (MMT-DY) processes with polarized $p$ and D beams Extraction of unknown PDFs from J/u production processes

Spin effects in baryon and meson productions.

Studies of spin effects in various exclusive reactions

Diffractive processes studies

Cross sections and double spin asymmetries in clastic reactions Spectroscopy of quarkoniums with any avallable decay modes

\section{On the Spin Program at NICA}

\section{Preliminary estimations of the MMT-DY processes feasibility (ffrst stage)}

DY cross sections (nb) in comparison with PAX (GSI, FAIR) and possibility to increase the statistics (month of data taking)

\begin{tabular}{||c|c|c|c|}
\hline & $\sigma_{D Y}$ total, nb & $L, \mathrm{~cm}^{-2} \mathrm{~s}^{-1}$ & K events \\
\hline PAX, $\sqrt{s}=14.6 \mathrm{GeV}$ & $\sim 2$ & $\sim 10^{30}$ & $\sim 10$ \\
NICA $\sqrt{s}=20 \mathrm{GeV}$ & $\sim 1$ & $\sim 10^{30}$ & $\sim 5$ \\
NICA $\sqrt{s}=26 \mathrm{GeV}$ & $\sim 1.3$ & $\sim 10^{30}$ & $\sim 7$ \\
\hline
\end{tabular}

\begin{tabular}{|c|c|c|c|c|c|c|}
\hline cut on $Q, \mathrm{GeV}$ & 1.5 & 1.6 & 1.7 & 1.8 & 1.9 & 2.0 \\
\hline \multicolumn{8}{|c|}{ NICA, $\sqrt{s}=20 \mathrm{GeV}$} \\
N events for a month, K & 14.1 & 10.5 & 8.8 & 7.3 & 6.1 & 5 \\
\hline \multicolumn{8}{|c|}{ NICA, $\sqrt{s}=26 \mathrm{GeV}$} \\
\hline$\sigma_{D Y}$ total,nb & 3.3 & 2.7 & 2.3 & 1.9 & 1.6 & 1.3 \\
N events for a month, K & 18 & 15 & 13 & 10 & 9 & 7 \\
\hline$\sigma_{D Y}$ total,nb & PAX, $\sqrt{s}=14.6 \mathrm{GeV}$ \\
\hline N events for a month, K & 24.4 & 20.7 & 16.7 & 13.9 & 11.8 & 10 \\
\hline
\end{tabular}

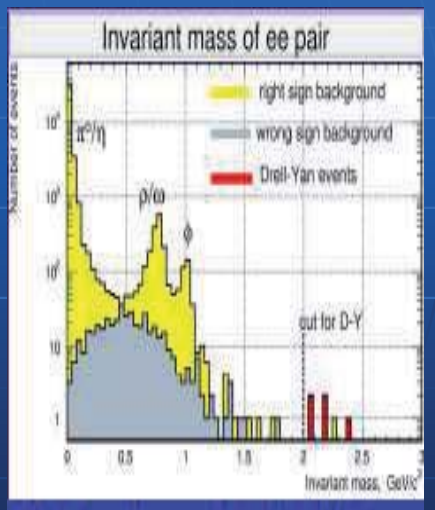

PAX background estimations 


\section{On the Spin Program at NICA}

Preliminary estimations of $\mathrm{J} / \Psi$ statistics in comparison with MMT-DY statistics

\begin{tabular}{|c|c|c|c|c|c|}
\hline$\sqrt{s}, \mathrm{GeV}$ & 20 & 26 & $\sqrt{3}, \mathrm{GeV}$ & 20 & 26 \\
\hline$\sigma_{J / \psi^{\prime}} B_{e^{+} e^{-}}, \mathrm{nb}$ & 10 & 16 & $\sigma_{D Y}, \mathrm{nb}$ & 0.9 & 1.3 \\
\hline N events for a month, K & 55 & 88 & N events for a month, K & 5 & 7 \\
\hline
\end{tabular}

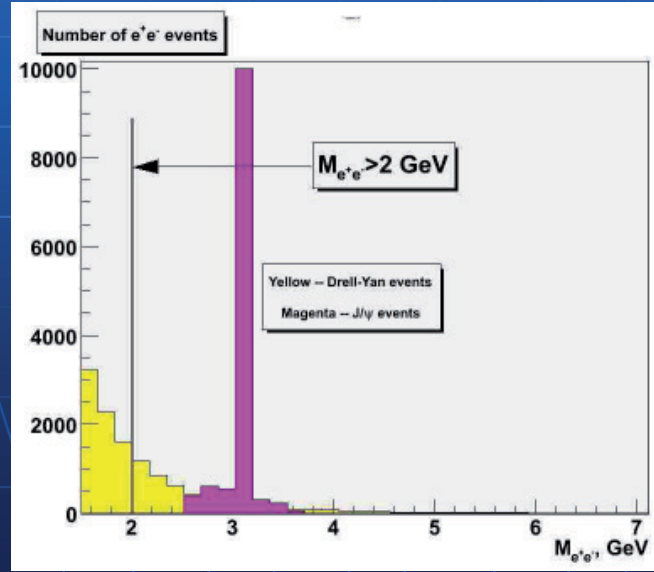

\section{Experiments on DY measurements}

\section{Experiment}

E615

NA10

E886

RHIC

PAX

COMPASS

J-PARC

SPASCHARM

NICA

\section{Status}

Finished

Finished

Running

Running

Plan > 2016

Plan > 2010

Plan > 2011

Plan?

Plan 2014

\section{Remarks}

Only unpolarized MMT-DY

Only unpolarized MMT-DY

Only unpolarized MMT-DY

Detector upgrade for MMT-DY measurements (collider)

Problem with $\bar{p}$ polarization (collider)

Only valence PDFs

low s (60-100 GeV²), only unpolarized proton beam

$\mathrm{s} \sim 140 \mathrm{GeV}^{2}$ for unpolarized proton beam $\mathrm{s} \sim 670 \mathrm{GeV}^{2}$ for polarized proton beams, high luminosity (collider) 


\section{Preliminary scheme of the experimental set-up for MMT-DY and J/ $\Psi$ measurements}

Important advantages of the detector:

2) $4 \pi$ geometry -increase of DY statistics

3) Minimal $X_{0}$ - effective detection of $\mathrm{e}+\mathrm{e}$ - pairs

3) Good angular resolution - very important for azimuthal spin asymmetries measurements in the wide kinematical region

Main parts of the detector (preliminarily):

- Silicon or Micromega (inner tracking)

- Drift chambers or straw (for tracking)

Cherenkov counter (for PID and trigger)

- EM calorimeter

- Trigger counters

- EndCap detectors

Similarily to the PAX set-up (hep-ex/0505054)

Set-up for muon pairs detection is also under consiveration
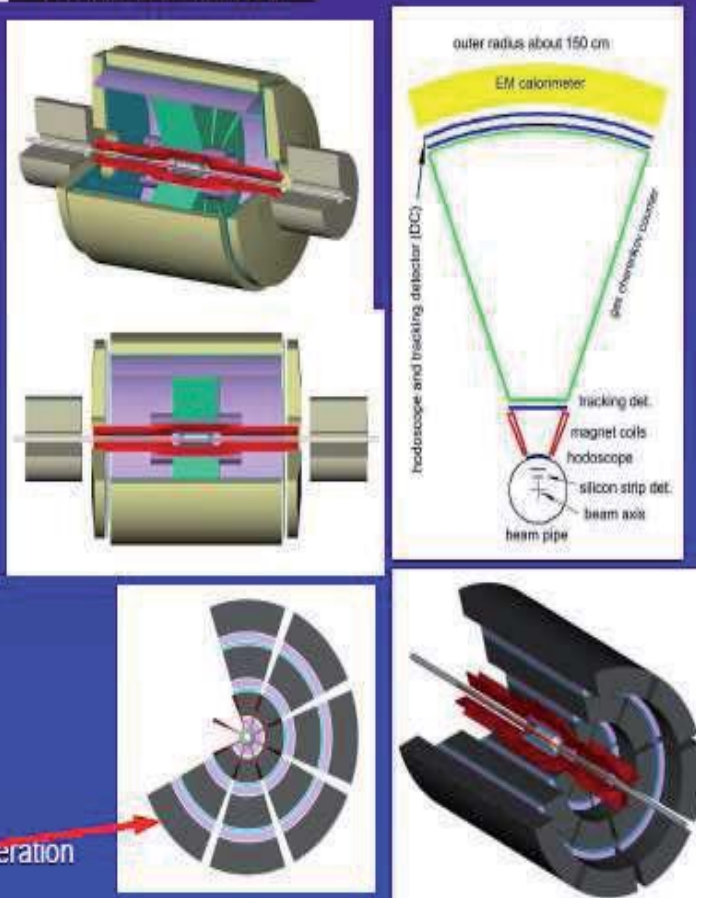

\section{Applied research at NICA}

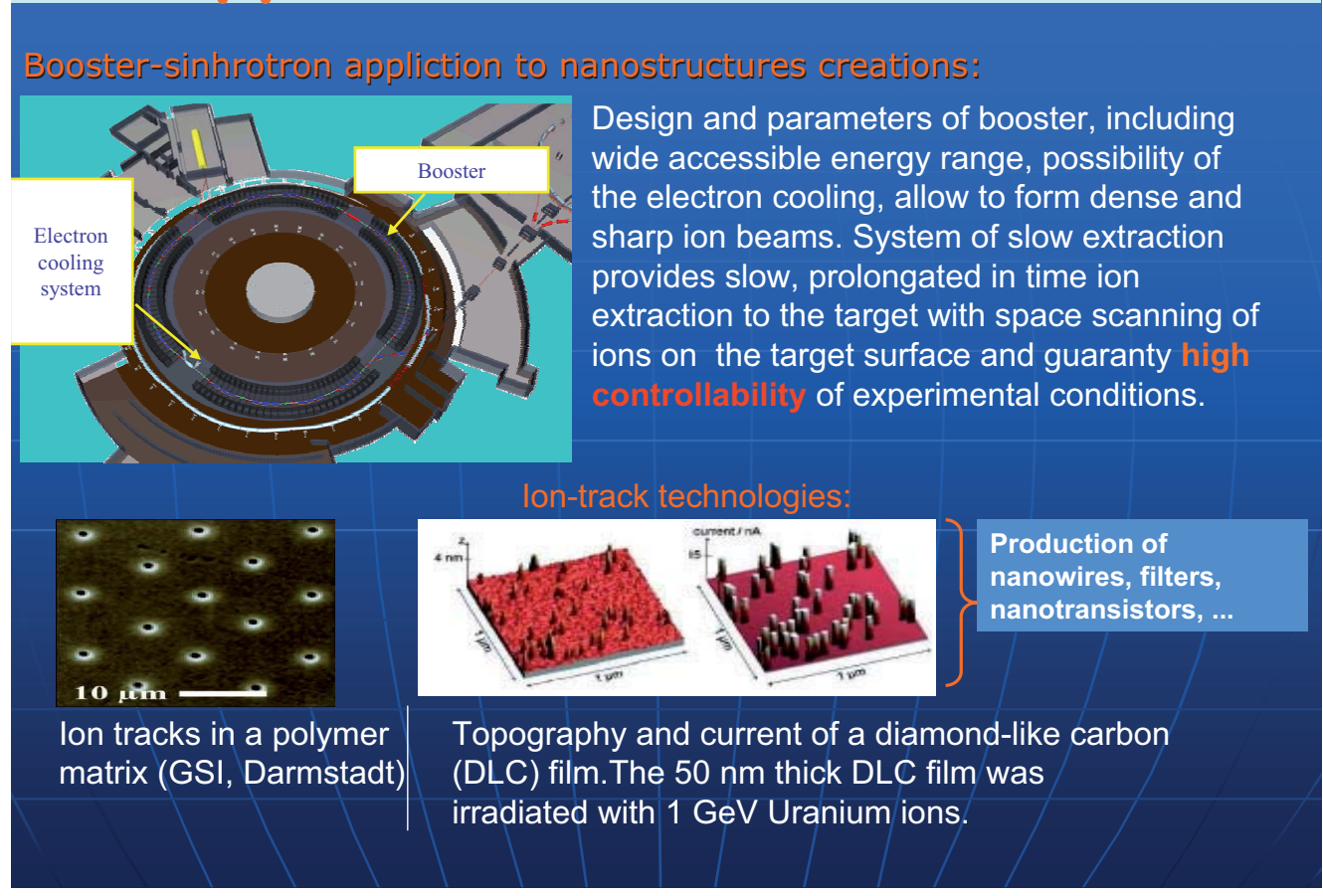




\section{Further realization of NICA project,}

as to both theory and MPD setup construction, suffers a deficit of qualified people, young scientists and engineers. The planned steps:

- More close collaboration with Russian institutes and JINR Member States

- Strengthening the relations with FAIR, RHIC and CERN where similar works are carried out (MoU)

- Profiling students of the Dubna University with orientation towards NICA/MPD problems

- Organization of student Schools and Meetings for young scientists on problems of NICA/MPD

\section{Round Table Discussions}

\section{Round Table Discussion I}

Searching for the mixed phase of strongly interacting matter at the JINR Nuclotron

July 7 - 9, 2005

http://theor.jinr.ru/meetings/2005/roundtable/

Round Table Discussion II

Searching for the mixed phase of strongly interacting matter at the JINR Nuclotron: Nuclotron facility development JINR, Dubna, October 6-7, 2006

http://theor.jinr.ru/meetings/2006/roundtable/

Round Table Discussion III

Searching for the mixed phase of strongly interacting

QCD matter at the NICA/MPD

JINR (Dubna), end 2008

http://nica.jinr.ru 


\section{Acknowledgements}

A. Efremov, V.Kekelidze, I.Meshkov,

G. Musulmanbekov, A. Nagaytsev,

O.Rogachevsky, I.Savin,

O.Shevchenko, V.Skokov,

O.Teryaev, V.Toneev,

NICA/MPD (SPD) working group

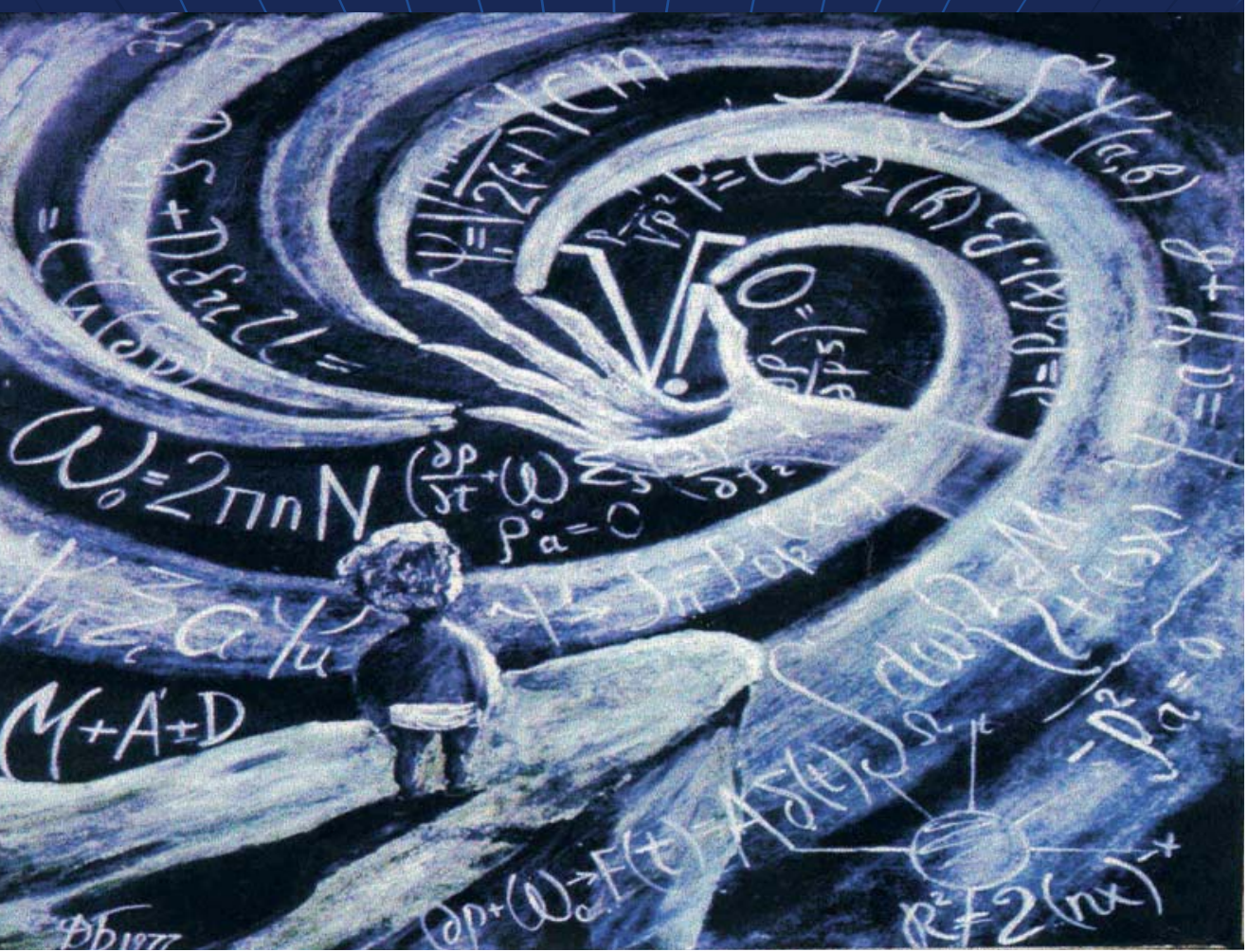




\section{Welcome to the} collaboration!
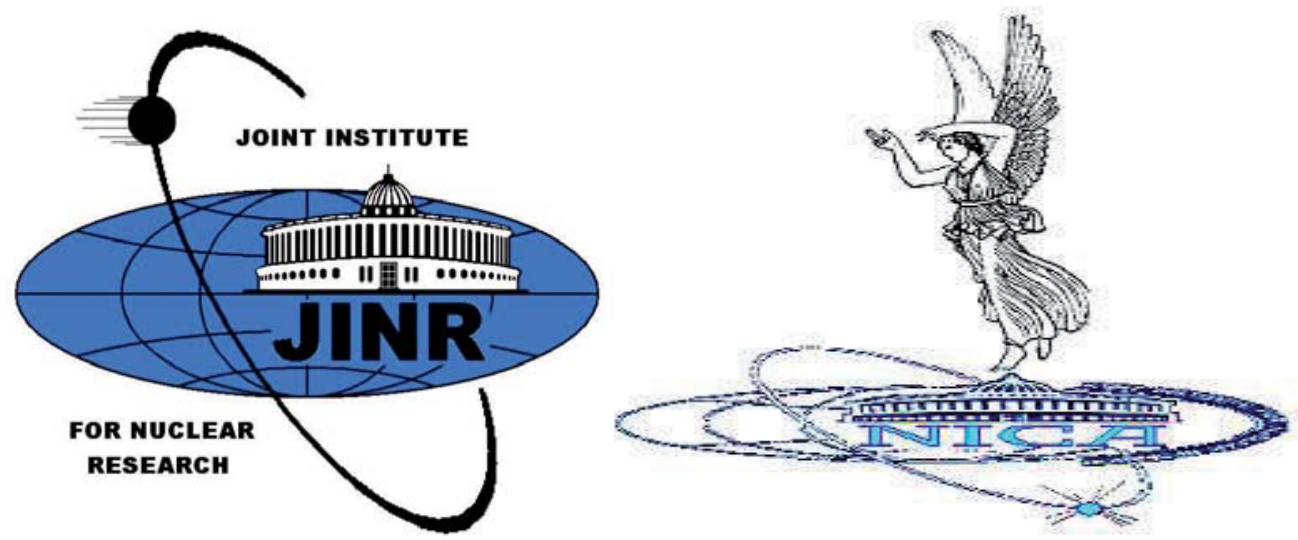

\section{Thank you for attention!}

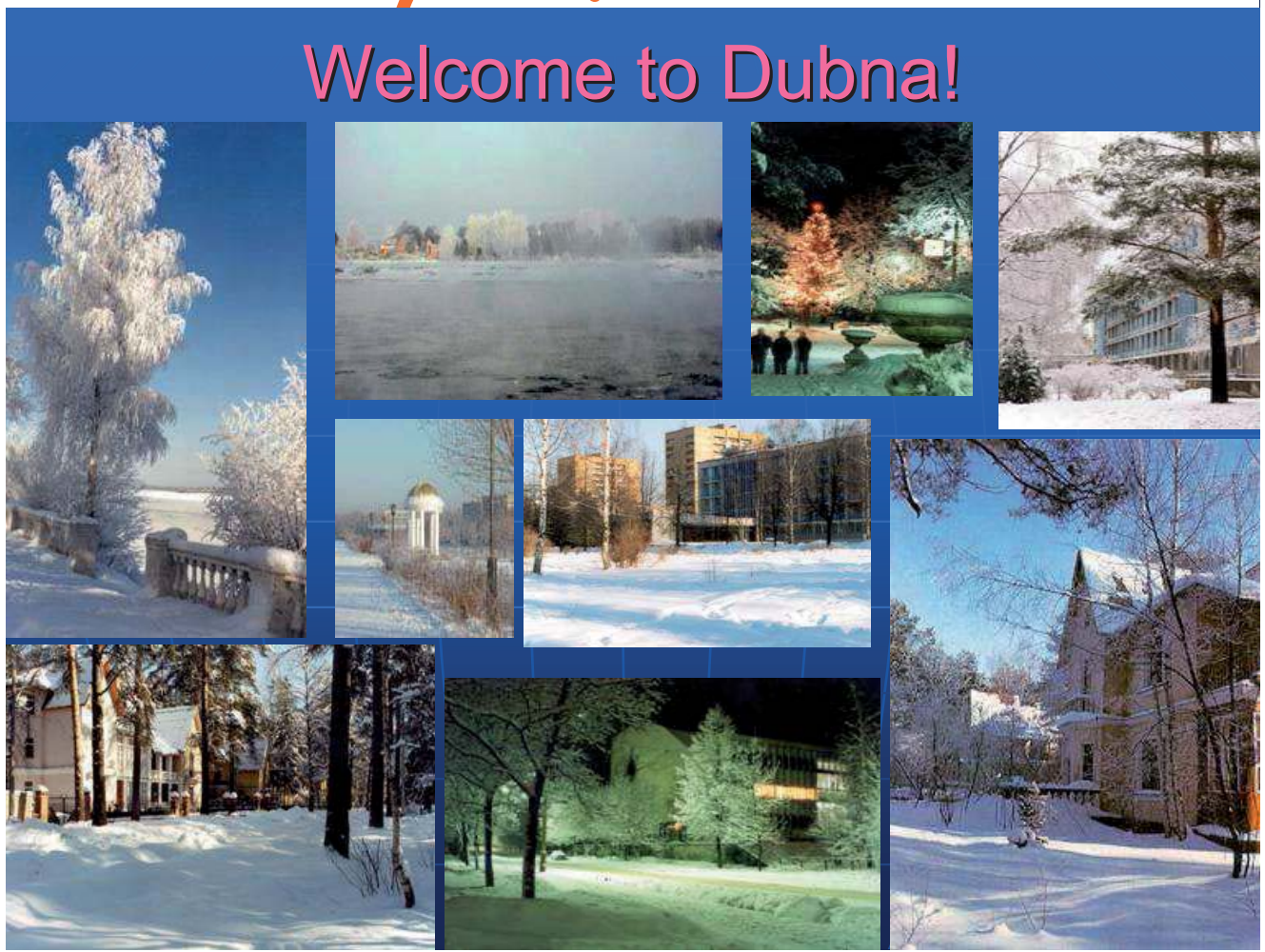

